\title{
A simple model system for the study of carbohydrate-aromatic interactions.
}

\section{Supplementary Material}

(19 pages)

Giancarlo Terraneo, ${ }^{\mathrm{a}}$ Donatella Potenza, ${ }^{\mathrm{a}}$ Angeles Canales ${ }^{\mathrm{b}}$,

Jesus Jiménez-Barbero, ${ }^{b *}$ Kim K. Baldridge, ${ }^{c^{*}}$ Anna Bernardi ${ }^{a *}$

a Universita' di Milano, Dipartimento di Chimica Organica e Industriale e Centro di Eccellenza CISI, via Venezian 21, 20133 Milano, Italy.

${ }^{b}$ Centro de Investigaciones Biológicas, Consejo Superior de Investigaciones Cientificas, Ramiro de Maeztu 9, 28040 Madrid, Spain,

${ }^{c}$ Institute of Organic Chemistry, University of Zürich, Winterthurerstrasse 190, CH-8057 Zürich, Switzerland 


\section{Synthesis of compounds $2 a-f$}

Diols 3a-b ${ }^{1}$ were initially converted to the stannylene derivative (dibutyltin oxide in benzene ${ }^{2}$ ) that was used without purification. The O-alkylation in position 4 was carried out using the triflate ${ }^{3} \mathbf{4}$ and CsF in DME (Scheme 1). ${ }^{4}$
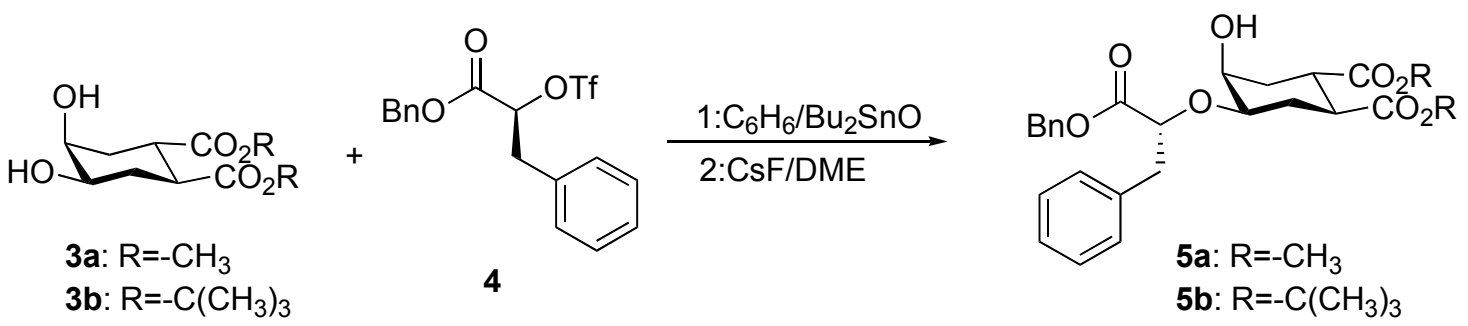

Scheme1. Synthesis compounds $\mathbf{5 a}$ and $\mathbf{5 b}$

Synthesis of 2a and 2b: the compound functionalized as a MOM ether (6) was synthesized, starting from 5a, using TBAI, DIPEA and methoxylmethylchloride, ${ }^{5}$ then the benzyl esters were removed using hydrogenation catalyzed by palladium, obtaining the carboxyl series $\mathbf{2 a - 2 b}$. (Scheme 2).

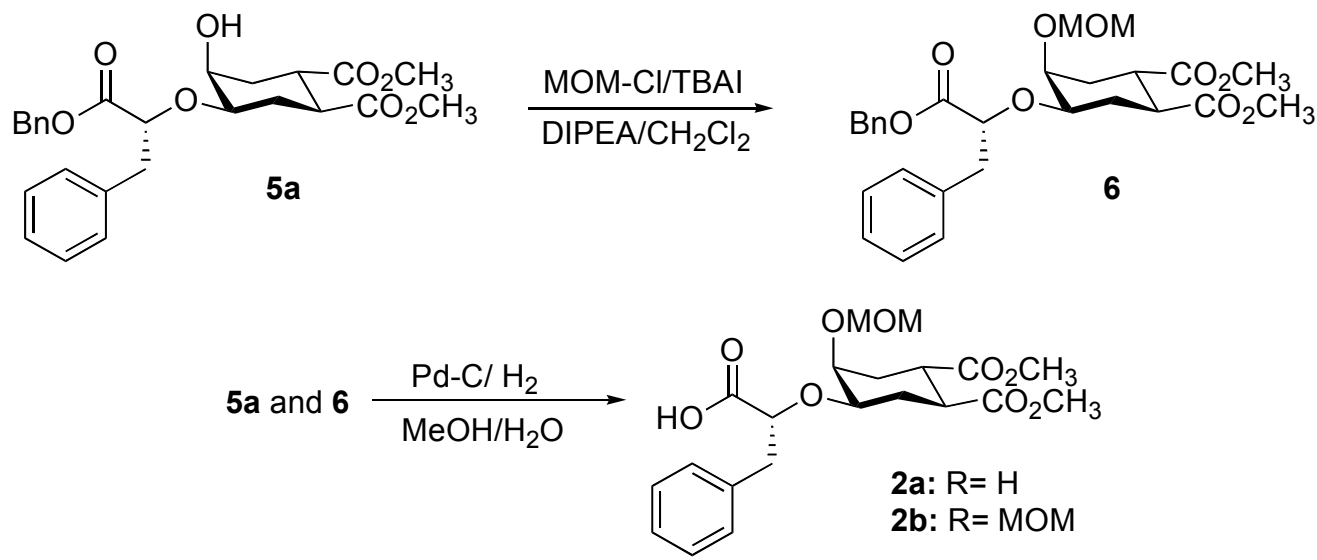

Scheme 2. Synthesis of compounds $2 \mathbf{a}$ and $\mathbf{2 b}$

For the synthesis of compounds $\mathbf{2 c - 2 f}$, we used a general precedure for the glycosylation reaction using as a donor the trichloroacetimidate of the protected sugars and as acceptor the monoether $\mathbf{5 b}$. The synthesis of the donors (7c-7d, Scheme 3) was carried out starting from the commercial unprotected sugars. Following penta-acetylation and hydrolysis of the anomeric position $\left(\mathrm{NH}_{2} \mathrm{NH}_{2} \mathrm{AcOH}\right)$ they were converted to the trichloroacetimidates using trichloroacetonitrile and DBU. The glycosylation reaction of the axial hydroxy group in position 5 was carried out at $-30{ }^{\circ} \mathrm{C}$ and catalyzed by trimethylsilyl triflate for $\mathbf{8 c}$ and $\mathbf{8 d}$ (Scheme 3). 
<smiles>CCOC(=O)C1CC(OC(Cc2ccccc2)C(=O)OBr)CC(O)C1C(=O)OCc1ccccc1</smiles>

$5 b$
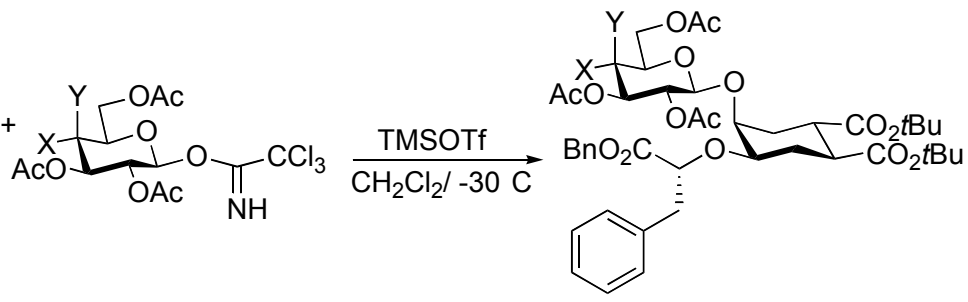

7c $(\mathrm{Glc}): \mathrm{X}=\mathrm{OAc} \mathrm{Y}=\mathrm{H}$ 7d (Gal): $X=H Y=O A c$
$8 \mathrm{c}=\mathrm{Glc}: \mathrm{X}=\mathrm{OAc} \mathrm{Y}=\mathrm{H}$ 8d $=\mathrm{Gal}: X=\mathrm{H} Y=O A c$

Scheme 3. General procedure for the glycosylation reaction for Glc and Gal derivatives

Donor 7e (Scheme 4) was synthesized by the same procedure from commercially available glucosamine. The glycosylation reaction was performed using triflic acid as catalyst at room temperature and then refluxed overnight (Scheme 4).

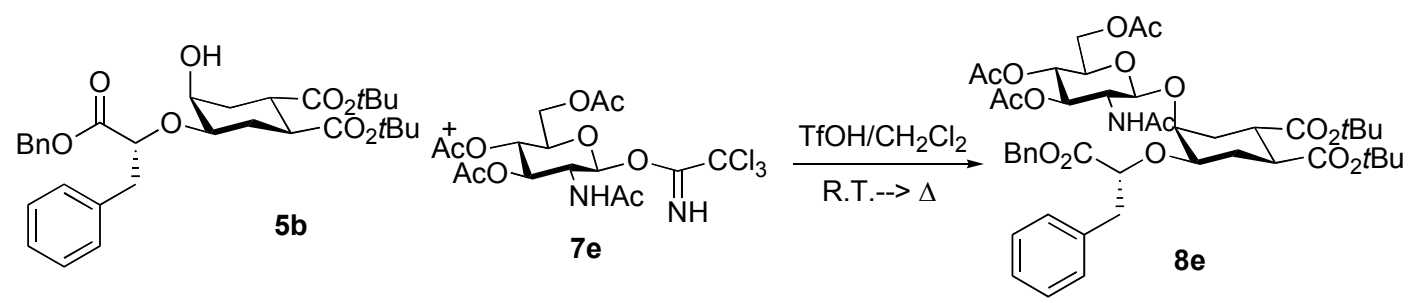

Scheme 4. General procedure for the glycosylation reaction for the GlcNAc derivative

The donor 7f (Scheme 5) was obtained from the corresponding protected N-acetyl-galactosamine ${ }^{6}$ The glycosylation was performed at $40^{\circ} \mathrm{C}$ using triflic acid as catalyst.

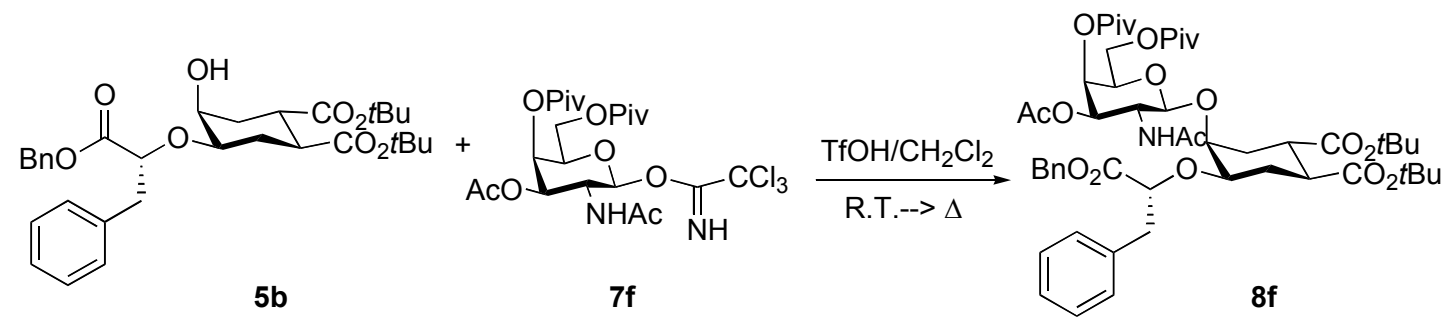

Scheme 5. General procedure for the glycosylation reaction for the GalNAc derivate

For the entire series the benzyl ester was removed using $\mathrm{H}_{2} / \mathrm{Pd}-\mathrm{C}$, obtaining the acids 9c-9f. (Scheme $6)$. 
8c, 8d and 8e, 8f $\frac{\mathrm{Pd}-\mathrm{C} / \mathrm{H}_{2}}{\mathrm{MeOH} / \mathrm{H}_{2} \mathrm{O}}$

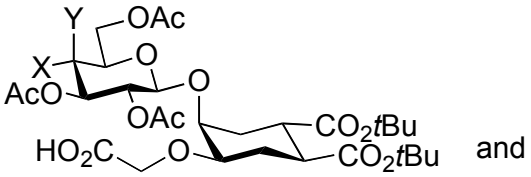

(

9c $=$ Glc: $X=O A c Y=H$

9d $=$ Gal: $X=H Y=O A c$
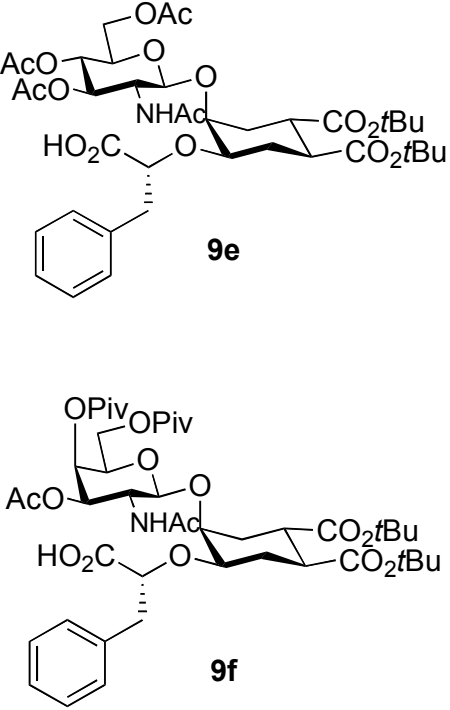

Scheme 6. General procedure for removing the benzyl ester

The removal of the acetyl groups and pivaloyl groups were carried out in standard condition ${ }^{7}$ using $\mathrm{MeONa} / \mathrm{MeOH}$ obtaining the final compounds 2c-2f. (Scheme 7)

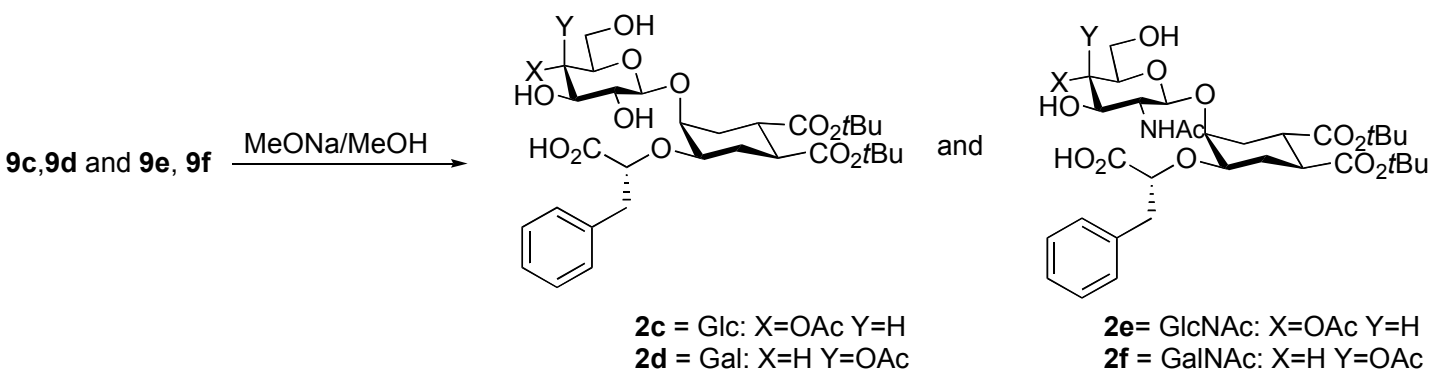

Scheme 7. General procedure for removal of the acetyl groups

\section{Experimental}

\section{General Procedures.}

Solvent were dried by standard procedures: dichloromethane and methanol were dried over calcium hydride; toluene, benzene, hexane and tetrahydrofurane were dried over sodium; N,Ndimethhylformamide and pyridine were dried over activated molecular sieves $4 \AA$. Reactions requiring anhydrous conditions were performed under agorn or nitrogen atmosphere. ${ }^{1} \mathrm{H}$ and ${ }^{13} \mathrm{C}$ spectra were treated al all times at 298-300 K, on Bruker AVANCE-500, Bruker AVANCE-400, Bruker AC-300 and AC-200 spectrometer. Chemical shift $(\delta)$ for ${ }^{1} \mathrm{H}$ and ${ }^{13} \mathrm{C}$ were expressed in ppm relative to internal $\left(\mathrm{CH}_{3}\right)_{4} \mathrm{Si}$ as standard. Signals were abbreviated with as $\mathrm{s}=$ singlet; $\mathrm{bs}=$ broad singlet; $\mathrm{d}=$ doublet; $\mathrm{t}=$ triplet; $\mathrm{q}=$ quartet; $\mathrm{m}=$ multiplet. For ${ }^{13} \mathrm{C}$-NMR spectra, only selected values are reported. Mass spectra were obtained with a VG 7070 EQ-HF $\left(\mathrm{FAB}^{+}\right)$apparatus. High resolution mass spectra (HRMS) were recorded on FT-ICR mass spectrometer APEX II - 4.7 T using as source (ESI). Electrospray Ionization (ESI) mass spectrometry experiments were carried out with Brucker Daltonics Esquire300plus, MALDI-TOF experiments were recorded Brucker Daltonics Omniflex. Optical rotations $[\alpha]_{\mathrm{D}}$ were measured in a cell of $1 \mathrm{dm}$ pathlength and $1 \mathrm{ml}$ capacity with Perkin-Elmeer 241 polarimeter. Thin layer 
chromatography (TLC) was carried out with precoated Merck $\mathrm{F}_{254}$ silica gel plates. Flash chromatography (FC) was carried out with Macherey-Nagel silica gel 60 (230-400 mesh) following general Still's procedure. (S)-Phenyllactic acid and its benzyl ester were purchased from Sigma-Aldrich. The synthesis of the diol $\mathbf{3 b}$ has been reported in ref. 1. The synthesis of triflate 4 is described in ref. 4 .

\section{Synthesis of (S)-3-Phenyl-2-trifluoromethanesulfonyloxy-propionic acid benzyl ester (4)}<smiles>O=C(OBr)C(CBr)Cc1ccccc1</smiles>

To a solution of $(S)$-phenyllactic acid benzyl ester $(1.50 \mathrm{~mL}, 5.85 \mathrm{mmol}, 1 \mathrm{eq})$ in dry $\mathrm{CH}_{2} \mathrm{Cl}_{2}(30 \mathrm{ml})$, trifluoromenthansulphonic anhydride $(1.06 \mathrm{~mL}, 6.43 \mathrm{mmol}, 1.1 \mathrm{eq})$ and 2,6 lutidine $(789 \mu \mathrm{L}, 6.79$ mmol, $1.16 \mathrm{eq}$ ) were added. The reaction mixture was stirred at room temperature for ca $20 \mathrm{~min}$. monitoring by TLC. After reaction completion the solvent was evaporated and the crude was purified by short pattern flash chromatography (Hexane/AcOEt 90:10) to give the product as colorless oil $(2.21 \mathrm{~g}$, 97\%). ${ }^{1} \mathrm{H}-\mathrm{NMR}\left(400 \mathrm{MHz}, \mathrm{CDCl}_{3}\right): 3.25\left(\mathrm{dd}, 1 \mathrm{H}, \mathrm{CH} \mathrm{HPh}, \mathrm{J}_{\mathrm{gem}}=14 \mathrm{~Hz}\right.$ and $\left.\mathrm{J}_{\mathrm{CHPh}-\mathrm{HL}}=7 \mathrm{~Hz}\right), 3.35$ (dd, $1 \mathrm{H}, \mathrm{CH} H \mathrm{Ph}, \mathrm{J}_{\mathrm{gem}}=14 \mathrm{~Hz}$ and $\left.\mathrm{J}_{\mathrm{CHPh}-\mathrm{HL}}=4 \mathrm{~Hz}\right), 5.25\left(\mathrm{~s}, 2 \mathrm{H}, \mathrm{CH}_{2} \mathrm{Ph}(\mathrm{Bn})\right), 5.25-5.35\left(\mathrm{~m}, 1 \mathrm{H}, \mathrm{H}_{\mathrm{L}}\right), 7.10-$ 7.40 (m, 10H, Har) ppm.

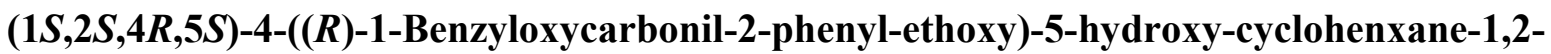 dicarboxyl acid dimethyl ester (5a)}<smiles>COC(=O)C1C(O)CC(OC(Cc2ccccc2)C(=O)O)CC1C(=O)O</smiles>

A solution of diol (3a) (600 mg, $2.58 \mathrm{mmol}$, 1eq) and $\mathrm{Bu}_{2} \mathrm{SnO}$ (643 mg, $\left.2.58 \mathrm{mmol}, 1 \mathrm{eq}\right)$, in dry benzene $(25 \mathrm{~mL})$, was refluxed for $8 \mathrm{~h}$, under $\mathrm{N}_{2}$, while the water was removed continuously. After this time benzene was evaporated under reduced pressure. The residue was redissolved in dry DME ( $8 \mathrm{~mL})$, under $\mathrm{N}_{2}$, and a solution of triflate $4(1.5 \mathrm{~g}, 3.87 \mathrm{mmol}, 1.5 \mathrm{eq})$ in dry DME $(8 \mathrm{~mL})$ was added. In the end CsF (588 mg, $3.87 \mathrm{mmol}, 1.5 \mathrm{eq}$ ) was added to the mixture. The reaction mixture was stirred at room temperature for $18 \mathrm{~h}$, monitoring by TLC (hexane/AcOEt 70:30). After reaction completion, $\mathrm{Et}_{2} \mathrm{O}$ was added and the organic phase was washed with $\mathrm{H}_{2} \mathrm{O}$. The organic phase, dried over $\mathrm{Na}_{2} \mathrm{SO}_{4}$, was evaporated and the crude was purified by flash chromatography on silica gel (Hexane/AcOEt 90:10). The compound was obtained as an amorphous white solid (400 mg, 33\%). $[\alpha]^{25}{ }_{\mathrm{D}}=+29.7 \mathrm{c}=0.8 \mathrm{CHCl}_{3}$; ${ }^{1} \mathrm{H}-\mathrm{NMR}\left(\mathrm{CDCl}_{3}, 400 \mathrm{MHz}\right): \delta 1.25-1.35\left(\mathrm{~m}, 1 \mathrm{H}, \mathrm{H}_{6 \mathrm{ax}}\right), 1.8\left(\mathrm{ddd}, 1 \mathrm{H}, \mathrm{H}_{3 \mathrm{ax}}, \mathrm{J}_{\mathrm{gem}}=\mathrm{J}_{3 \mathrm{ax}-4}=\mathrm{J}_{3 \mathrm{ax}-2}=12 \mathrm{~Hz}\right)$, 2.05-2.18 (m, $2 \mathrm{H}, \mathrm{H}_{6 \mathrm{eq}}$ and $\left.\mathrm{H}_{3 \mathrm{ax}}\right), 2.38\left(\mathrm{td}, 1 \mathrm{H}, \mathrm{H}_{6}, \mathrm{~J}_{6-3 \mathrm{ax}}=\mathrm{J}_{6-1}=12 \mathrm{~Hz}\right.$ and $\left.\mathrm{J}_{2-3 \mathrm{eq}}=4 \mathrm{~Hz}\right), 2.85-2.96(\mathrm{~m}, 2 \mathrm{H}$, $\mathrm{C} H \mathrm{HPh}$ and $\left.\mathrm{H}_{1}\right), 3.15\left(\mathrm{dd}, 1 \mathrm{H}, \mathrm{CH} H \mathrm{Ph}, \mathrm{J}_{\mathrm{gem}}=14 \mathrm{~Hz} \mathrm{~J}_{\mathrm{CHPh}-\mathrm{HL}}=3.2 \mathrm{~Hz}\right), 3.25-3.30\left(\mathrm{~m}, 1 \mathrm{H}, \mathrm{H}_{4}\right), 3.60(\mathrm{bs}$, $\left.1 \mathrm{H}, \mathrm{H}_{5}\right), 3.63\left(\mathrm{~s}, 3 \mathrm{H}, \mathrm{OCH}_{3}\right), 3.68\left(\mathrm{~s}, 3 \mathrm{H}, \mathrm{OCH}_{3}\right), 4.18\left(\mathrm{dd}, 1 \mathrm{H}, \mathrm{H}_{\mathrm{L}}, \mathrm{J}_{\mathrm{HL}-\mathrm{CH} 2 \mathrm{Ph}}=3.2\right.$ and $\left.14 \mathrm{~Hz}\right), 5.20(\mathrm{~d}$, $\left.1 \mathrm{H}, \mathrm{OCHHPh}(\mathrm{Bn}), \mathrm{J}_{\mathrm{gem}}=12 \mathrm{~Hz}\right), 5.30\left(\mathrm{~d}, 1 \mathrm{H}, \mathrm{OCH} H \mathrm{Ph}(\mathrm{Bn}), \mathrm{J}_{\mathrm{gem}}=12 \mathrm{~Hz}\right), 7.15-7.45\left(\mathrm{~m}, 5 \mathrm{H}, \mathrm{H}_{\mathrm{ar}}\right)$ ppm; ${ }^{13} \mathrm{C}-\mathrm{NMR}\left(\mathrm{CDCl}_{3}, 100.6 \mathrm{MHz}\right): \delta 27.83,32.04,37.93,39.14,43.13,51.86,51.89,63.90,67.10$, 77.61, 77.91, 127.32, 128.46, 128.46, 128.63, 128.73, 128.88, 129.13, 135.29, 137.32, 171.63, 173.73, 175.32 ppm; MS (F.A.B. ${ }^{+}$): $471[\mathrm{MH}]^{+}$ 


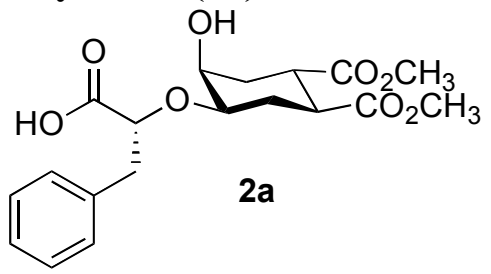

To a solution of compound 5a (20 mg, $0.042 \mathrm{mmol}, 1 \mathrm{eq})$ in $\mathrm{MeOH} / \mathrm{H}_{2} \mathrm{O}$ 90:10 (0.5 mL), a catalytic amount of $\mathrm{Pd}-\mathrm{C} 10 \%$ was added. The mixture was stirred under $\mathrm{H}_{2}$ atmosphere at room temperature for $3 \mathrm{~h}$, monitoring by TLC $\left(\mathrm{CHCl}_{3} / \mathrm{MeOH}\right.$ : $95: 5$ and $\left.1 \% \mathrm{CH}_{3} \mathrm{COOH}\right)$. After reaction completion, the mixture was filtered through a celite pad, washing several times with methanol. The collected organic phase was evaporated under reduced pressure. The compound was obtained as an amorphous white solid $(15 \mathrm{mg}, 95 \%)$. $[\alpha]^{25}{ }_{\mathrm{D}}=+24.5 \mathrm{c}=1.0 \mathrm{CHCl}_{3} ;{ }^{1} \mathrm{H}-\mathrm{NMR}\left(\mathrm{D}_{2} \mathrm{O}, 400 \mathrm{MHz}\right): \delta 1.45-1.55\left(\mathrm{~m}, 1 \mathrm{H}, \mathrm{H}_{6 \mathrm{ax}}\right)$, $1.68\left(\mathrm{ddd}, 1 \mathrm{H}, \mathrm{H}_{3 \mathrm{ax}}, \mathrm{J}_{\mathrm{gem}}=\mathrm{J}_{3 \mathrm{ax}-4}=\mathrm{J}_{3 \mathrm{ax}-2}=12 \mathrm{~Hz}\right), 1.98-2.07\left(\mathrm{~m}, 2 \mathrm{H}, \mathrm{H}_{6 \mathrm{eq}}\right.$ and $\left.\mathrm{H}_{3 \mathrm{eq}}\right), 2.68\left(\mathrm{td}, 1 \mathrm{H}, \mathrm{H}_{2}, \mathrm{~J}_{2-}\right.$ ${ }_{3 a x}=J_{2-1}=12 \mathrm{~Hz}$ and $\left.J_{2-3 e q}=4 \mathrm{~Hz}\right), 2.82\left(\mathrm{td}, 1 \mathrm{H}, \mathrm{H}_{1}, \mathrm{~J}_{1-2}=\mathrm{J}_{1-6 \mathrm{ax}}=12 \mathrm{~Hz}\right.$ and $\left.\mathrm{J}_{1-6 e q}=4 \mathrm{~Hz}\right), 2.90(\mathrm{dd}, 1 \mathrm{H}$, $\mathrm{CHHPh}, \mathrm{J}_{\mathrm{gem}}=14 \mathrm{~Hz}$ and $\left.\mathrm{J}_{\mathrm{CHPh}-\mathrm{HL}}=9 \mathrm{~Hz}\right), 3.10\left(\mathrm{dd}, 1 \mathrm{H}, \mathrm{CH} H \mathrm{Ph}, \mathrm{J}_{\mathrm{gem}}=14 \mathrm{~Hz}\right.$ and $\left.\mathrm{J}_{\mathrm{CHPh}-\mathrm{HL}}=5 \mathrm{~Hz}\right)$, 3.40-3.47 (m, 1H, $\left.\mathrm{H}_{4}\right), 3.62\left(\mathrm{~s}, 3 \mathrm{H},-\mathrm{OCH}_{3}\right), 3.66\left(\mathrm{~s}, 3 \mathrm{H},-\mathrm{OCH}_{3}\right), 3.85\left(\mathrm{bs}, 1 \mathrm{H}, \mathrm{H}_{5}\right), 4.35\left(\mathrm{dd}, 1 \mathrm{H}, \mathrm{H}_{\mathrm{L}}\right.$, $\mathrm{J}_{\mathrm{HL}-\mathrm{CH} 2 \mathrm{Ph}}=5$ and $\left.9 \mathrm{~Hz}\right), 7.20-7.35\left(\mathrm{~m}, 5 \mathrm{H}, \mathrm{H}_{\mathrm{ar}}\right) \mathrm{ppm} ;{ }^{13} \mathrm{C}-\mathrm{NMR}\left(\mathrm{D}_{2} \mathrm{O}, 50.3 \mathrm{MHz}\right): \delta 23.02,29.78,34.62$, 41.09, 41.21, 45.61, 55.34, 67.37, 80.12, 80.63, 129.78, 131.40, 132.06, 139.74, 179.00, 179.39, 180.02 ppm; HRMS (ESI) $\mathrm{C}_{19} \mathrm{H}_{24} \mathrm{O}_{8}$ calcd. [M-H]: 379.13984 found [M-H] $: 379.13835$

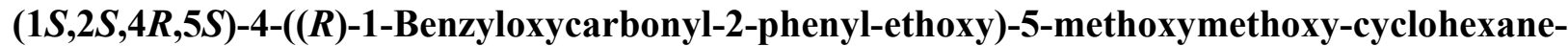
1,2-dicarboxyl acid methyl ester (6)<smiles>COC(=O)C1CC(OC(Cc2ccccc2)C(=O)OCc2ccccc2)CC(C(=O)OC)C1C(=O)OC</smiles>

A solution of $5 \mathrm{a}$ (30 mg, $0.064 \mathrm{mmol}, 1 \mathrm{eq})$ and TBAI $(5 \mathrm{mg}, 0.013 \mathrm{mmol}, 0.2 \mathrm{eq})$ in $\mathrm{CH}_{2} \mathrm{Cl}_{2}(0.5 \mathrm{~mL})$ dry was cooled at $0^{\circ} \mathrm{C}$ then DIPEA $(24.1 \mu \mathrm{L}, 0.141 \mathrm{mmol}, 2.2 \mathrm{eq})$ and methoxylmethylchloride $(10 \mu \mathrm{L}$, $0.128 \mathrm{mmol}, 2.0 \mathrm{eq}$ ) were added in it. The reaction mixture was stirred a R.T. monitoring by TLC (petroleum ether/AcOEt 70:30), after $3 \mathrm{~h}$ another aliquot of DIPEA and MOMCl were added. The reaction mixture was stirred at R.T. temperature overnight. After reaction completion, the mixture was washed with water, a solution of $\mathrm{HCl} 5 \%$ and water. The organic phase was dried over $\mathrm{Na}_{2} \mathrm{SO}_{4}$ and evaporated under reduce pressure. The crude was purified by flash chromatography on silica gel (petroleum ether/AcOEt 70:30) obtaining the product as colorless oil $(13 \mathrm{mg}, 40 \%)$. $[\alpha]^{25}=8.40 \mathrm{c}=1.2$ $\mathrm{CHCl}_{3} ;{ }^{1} \mathrm{H}-\mathrm{NMR}\left(\mathrm{CDCl}_{3}, 400 \mathrm{MHz}\right): 1.20-1.40\left(\mathrm{~m}, 1 \mathrm{H}, \mathrm{H}_{6 \mathrm{ax}}\right), 1.90\left(\mathrm{ddd}, 1 \mathrm{H}, \mathrm{H}_{3 \mathrm{ax}}, \mathrm{J}_{\mathrm{gem}}=\mathrm{J}_{3 \mathrm{ax}-4}=\mathrm{J}_{3 \mathrm{ax}-2}=12\right.$ $\mathrm{Hz}), 2.05-2.20\left(\mathrm{~m}, 2 \mathrm{H}, \mathrm{H}_{6 \mathrm{eq}}\right.$ and $\left.\mathrm{H}_{3 \mathrm{eq}}\right), 2.55-2.65\left(\mathrm{~m}, 1 \mathrm{H}, \mathrm{H}_{2}\right)$ 2.90-3.12 (m, 3H, $\mathrm{CH}_{2} \mathrm{Ph}$ and $\left.\mathrm{H}_{1}\right), 3.15-$ $3.25\left(\mathrm{~m}, 1 \mathrm{H}, \mathrm{H}_{4}\right), 3.45\left(\mathrm{~s}, 3 \mathrm{H}, \mathrm{CH}_{3} \mathrm{O}-\right), 3.67\left(\mathrm{~s}, 3 \mathrm{H},-\mathrm{OCH}_{3}\right), 3.68\left(\mathrm{~s}, 3 \mathrm{H},-\mathrm{OCH}_{3}\right), 3.75\left(\mathrm{bs}, 1 \mathrm{H}, \mathrm{H}_{5}\right)$, 4.18-4.30 (m, 2H, $\mathrm{H}_{\mathrm{L}}$ and $\left.\mathrm{CHHO}_{\mathrm{MOM}}\right), 4.45\left(\mathrm{~d}, 1 \mathrm{H}, \mathrm{CH} H \mathrm{O}_{\mathrm{MOM}}, \mathrm{J}_{\mathrm{gem}}=7 \mathrm{~Hz}\right), 5.13(\mathrm{~d}, 1 \mathrm{H}, \mathrm{OCH} \mathrm{HPh}$ $\left.(\mathrm{Bn}), \mathrm{J}_{\mathrm{gem}}=12 \mathrm{~Hz}\right), 5.18\left(\mathrm{~d}, 1 \mathrm{H}, \mathrm{OCH} H \mathrm{Ph}(\mathrm{Bn}), \mathrm{J}_{\mathrm{gem}}=12 \mathrm{~Hz}\right), 7.15-7.45\left(\mathrm{~m}, 10 \mathrm{H}, \mathrm{H}_{\mathrm{ar}}\right) \mathrm{ppm} ;{ }^{13} \mathrm{C}-\mathrm{NMR}-$ HETCOR $\left(\mathrm{CDCl}_{3}, 400 \mathrm{MHz}\right): 29.30,33.34,39.61,40.42,43.66,52.55,56.19,67.31,71.56,78.84$, 79.44, 97.04, 126.98, 129.38, 129.81 ppm; MS (ESI): $553.1[\mathrm{M}+\mathrm{K}]^{+}, 537.1[\mathrm{M}+\mathrm{Na}]^{+}$ 


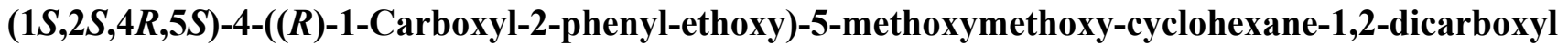
acid methyl ester (2b)<smiles>COC(=O)C1CC(OC(Cc2ccccc2)C(=O)O)CC(C(=O)OC)C1C(C)=O</smiles>

To a solution of $6(13 \mathrm{mg}, 0.025 \mathrm{mmol}, 1 \mathrm{eq})$ in $\mathrm{MeOH} / \mathrm{H}_{2} \mathrm{O} 90: 10(0.5 \mathrm{~mL})$ a catalytic amount of Pd-C $10 \%$ was added. The mixture was stirred under $\mathrm{H}_{2}$ atmosphere at room temperature, monitoring by TLC $\left(\mathrm{CHCl}_{3} / \mathrm{MeOH}: 90: 10\right)$. After $6 \mathrm{~h}$, the mixture was filtered through a celite pad, washing several times with methanol. The collected organic phase was evaporated under reduced pressure and the crude was purified by flash chromatography on silica gel $\left(\mathrm{CHCl}_{3} / \mathrm{MeOH}\right.$ : 90:10) obtaining the product as amorphous white solid $(8 \mathrm{mg}, 80 \%)$. $[\alpha]^{25}=+12.8 \mathrm{c}=0.4 \mathrm{CHCl}_{3} ;{ }^{1} \mathrm{H}-\mathrm{NMR}\left(\mathrm{D}_{2} \mathrm{O}, 400 \mathrm{MHz}\right): 1.40-1.50$ $\left(\mathrm{m}, 1 \mathrm{H}, \mathrm{H}_{6 \mathrm{ax}}\right), 1.68$ (ddd, $1 \mathrm{H}, \mathrm{H}_{3 \mathrm{ax}}, \mathrm{J}_{\text {gem }}=\mathrm{J}_{3 \mathrm{ax}-4}=\mathrm{J}_{3 \mathrm{ax}-2}=12 \mathrm{~Hz}$ ), 2.05-2.17 (m, 2H, $\mathrm{H}_{6 \mathrm{eq}}$ and $\mathrm{H}_{3 \mathrm{eq}}$ ), 2.70$2.85\left(\mathrm{~m}, 3 \mathrm{H}, \mathrm{H}_{2}, \mathrm{CHHPh}\right.$ and $\left.\mathrm{H}_{1}\right), 3.05\left(\mathrm{dd}, 1 \mathrm{H}, \mathrm{CH} H \mathrm{Ph}, \mathrm{J}_{\mathrm{gem}}=14 \mathrm{~Hz}\right.$ and $\left.\mathrm{J}_{\mathrm{CHPh}-\mathrm{HL}}=4 \mathrm{~Hz}\right), 3.23(\mathrm{~s}, 3 \mathrm{H}$, -

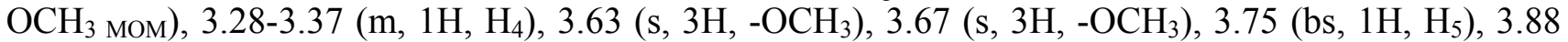
$\left(\mathrm{d}, 1 \mathrm{H}, \mathrm{CH} \mathrm{HO}_{\mathrm{MOM}}, \mathrm{J}_{\mathrm{gem}}=7 \mathrm{~Hz}\right), 4.15\left(\mathrm{dd}, 1 \mathrm{H}, \mathrm{H}_{\mathrm{L}}, \mathrm{J}_{\mathrm{HL}-\mathrm{CHPh}}=9.5\right.$ and $\left.4 \mathrm{~Hz}\right), 4.25\left(\mathrm{~d}, 1 \mathrm{H}, \mathrm{CH} H \mathrm{O}_{\mathrm{MOM}}\right.$, $\left.\mathrm{J}_{\mathrm{gem}}=7 \mathrm{~Hz}\right), 7.20-7.40\left(\mathrm{~m}, 5 \mathrm{H}, \mathrm{H}_{\mathrm{ar}}\right) \mathrm{ppm} ;{ }^{13} \mathrm{C}-\mathrm{NMR}\left(\mathrm{D}_{2} \mathrm{O}, 100.6 \mathrm{MHz}\right): 28.42,31.36,39.09,43.12$, 52.64, 52.71, 55.24, 72.27, 77.09, 81.07, 96.07, 126.73, 128.61, 129.37, 138.22, 176.46, 177.32 ppm; HRMS (ESI) $\mathrm{C}_{21} \mathrm{H}_{28} \mathrm{O}_{9}$ calcd. [M-H] $: 423.16606$ found [M-H] $: 423.16453$

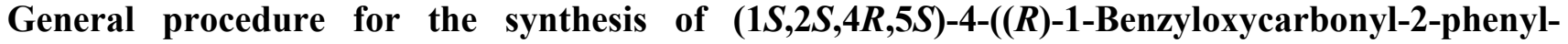
ethoxy)-5-(2,3,4,6-tetra- $O$-acetyl- $\beta$-D-glucopyranosyl)-cyclohexane-1,2-dicarboxyl acid di-tertbutyl ester $(8 \mathrm{c})$ and $(1 S, 2 S, 4 R, 5 S)-4-((R)-1-B e n z y l o x y c a r b o n y l-2-p h e n y l-e t h o x y)-5-(2,3,4,6$-tetra$O$-acetyl- $\beta$-D-galactopyranosyl)-cyclohexane-1,2-dicarboxyl acid di-tert-butyl ester (8d)

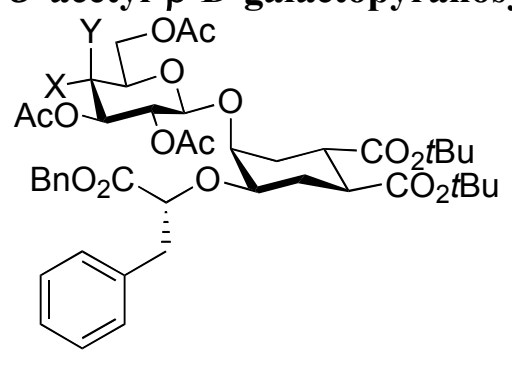

$8 \mathrm{c}=\mathrm{Glc}: \mathrm{X}=\mathrm{OAc} \mathrm{Y=H}$

8d $=$ Gal: $X=\mathrm{H} Y=\mathrm{OAc}$

To a solution of monoether $\mathbf{5 b}(50 \mathrm{mg}, 0.09 \mathrm{mmol}, 1 \mathrm{eq})$ and 2,3,4,6-tetra- $O$-acetyl-glucopyranosyl trichloroacetimidate (7c) or 2,3,4,6-tetra- $O$-acetyl-galactopyranosyl trichloroacetimidate (7d) $(129 \mathrm{mg}$, $0.27 \mathrm{mmol}, 3 \mathrm{eq})$ in dry $\mathrm{CH}_{2} \mathrm{Cl}_{2}(2 \mathrm{~mL})$, under $\mathrm{N}_{2}$ in presence of molecular sieves $4 \AA$ acid washed (150 $\mathrm{mg}$ ) was stirred for $10 \mathrm{~min}$ at $-30^{\circ} \mathrm{C}$ and after this time TMSOTf $(1.6 \mu \mathrm{L}, 0.009 \mathrm{mmol}, 0.1 \mathrm{eq})$ was dropped in it. The reaction mixture was stirred at $-30^{\circ} \mathrm{C}$ for $3 \mathrm{~h}$ monitoring by TLC (petroleum ether/AcOEt: 75:25). After reaction completion, TEA was added until $\mathrm{pH}=7$ was reached and the solvent was evaporated under reduced pressure. The crude product was purified by flash chromatography on silica gel (petroleum ether/AcOEt 75:25) obtaining the product as amorphous white solid.

8c: (yield: $36 \mathrm{mg}, 45 \%)[\alpha]^{25}=+10.8 \mathrm{c}=1 \mathrm{CHCl}_{3} ;{ }^{1} \mathrm{H}-\mathrm{NMR}\left(\mathrm{C}_{6} \mathrm{D}_{6}, 400 \mathrm{MHz}\right): 1.15-1.25\left(\mathrm{~m}, 1 \mathrm{H}, \mathrm{H}_{6 \mathrm{ax}}\right)$, $1.48\left(\mathrm{~s}, 9 \mathrm{H},\left(\mathrm{CH}_{3}\right)_{3} \mathrm{C}-\right), 1.53\left(\mathrm{~s}, 9 \mathrm{H},\left(\mathrm{CH}_{3}\right)_{3} \mathrm{C}-\right), 1.78\left(\mathrm{~s}, 3 \mathrm{H}, \mathrm{CH}_{3} \mathrm{CO}-\right), 1.80\left(\mathrm{~s}, 3 \mathrm{H}, \mathrm{CH}_{3} \mathrm{CO}-\right), 1.85(\mathrm{~s}$, $\left.3 \mathrm{H}, \mathrm{CH}_{3} \mathrm{CO}-\right), 2.05$ (s, 3H, $\left.\mathrm{CH}_{3} \mathrm{CO}-\right), 2.18$ (ddd, 1H, $\mathrm{H}_{3 \mathrm{ax}} \mathrm{J}_{\mathrm{gem}}=\mathrm{J}_{3 \mathrm{ax}-2}=\mathrm{J}_{3 \mathrm{ax}-4}=12 \mathrm{~Hz}$ ), 2.35 (dt, $1 \mathrm{H}, \mathrm{H}_{3 \mathrm{eq}}$, $\mathrm{Jgem}=12 \mathrm{~Hz}$ and $\left.\mathrm{J}_{3 \text { eq- }-2}=\mathrm{J}_{3 \mathrm{eq}-4}=4 \mathrm{~Hz}\right), 2.47\left(\mathrm{dt}, 1 \mathrm{H}, \mathrm{H}_{6 \mathrm{eq}} \mathrm{J}_{\text {gem }}=12 \mathrm{~Hz}\right.$ and $\left.\mathrm{J}_{6 \mathrm{eq}-1}=\mathrm{J}_{6 \mathrm{eq}-5}=4 \mathrm{~Hz}\right), 2.75(\mathrm{td}$, 
$1 \mathrm{H}, \mathrm{H}_{2}, \mathrm{~J}_{2-3 \mathrm{ax}}=\mathrm{J}_{2-1}=12 \mathrm{~Hz}$ and $\left.\mathrm{J}_{2-3 \mathrm{eq}}=4 \mathrm{~Hz}\right), 3.05\left(\mathrm{dd}, 1 \mathrm{H}, \mathrm{CHHPh} \mathrm{J}_{\mathrm{gem}}=14 \mathrm{~Hz}\right.$ and $\left.\mathrm{J}_{\mathrm{CHPh}-\mathrm{HL}}=10 \mathrm{~Hz}\right)$, 3.05-3.13 (m, 2H, $\mathrm{CH} H \mathrm{Ph}$ and $\left.\mathrm{H}_{4}\right), 3.25-3.35\left(\mathrm{~m}, 2 \mathrm{H}, \mathrm{H}_{1}\right.$ and $\left.\mathrm{GlcH}_{5}\right), 3.82\left(\mathrm{bs}, 1 \mathrm{H}, \mathrm{H}_{5}\right), 4.18-4.20(\mathrm{~m}$, $2 \mathrm{H}, \mathrm{GlcH}_{6}$ and $\left.\mathrm{H}_{\mathrm{L}}\right), 4.30-4.40\left(\mathrm{~m}, 2 \mathrm{H}, \mathrm{GlcH}_{1}\right.$ and $\left.\mathrm{GlcH}_{6}\right), 5.07\left(\mathrm{~d}, 1 \mathrm{H}, \mathrm{CH} \mathrm{HPh}(\mathrm{Bn}) \mathrm{J}_{\mathrm{gem}}=12 \mathrm{~Hz}\right), 5.13$ $\left(\mathrm{d}, 1 \mathrm{H}, \mathrm{CH} H \mathrm{Ph}(\mathrm{Bn}) \mathrm{J}_{\mathrm{gem}}=12 \mathrm{~Hz}\right), 5.20\left(\mathrm{t}, \mathrm{GlcH}_{2}, \mathrm{~J}_{\mathrm{GH} 2-\mathrm{GH} 3}=\mathrm{J}_{\mathrm{GH} 2-\mathrm{GH} 4}=9 \mathrm{~Hz}\right), 5.25\left(\mathrm{t}, \mathrm{GlcH}_{4}, \mathrm{~J}_{\mathrm{GH} 4-}\right.$ $\left.\mathrm{GH}_{3}=\mathrm{J}_{\mathrm{GH} 4-\mathrm{GH} 5}=9 \mathrm{~Hz}\right), 5.50\left(\mathrm{t}, \mathrm{GlcH}_{3}, \mathrm{~J}_{\mathrm{GH} 3-\mathrm{GH} 2}=\mathrm{J}_{\mathrm{GH} 3-\mathrm{GH} 4}=9 \mathrm{~Hz}\right), 7.25-7.45\left(\mathrm{~m}, 5 \mathrm{H}, \mathrm{H}_{\mathrm{ar}}\right) \mathrm{ppm} ;{ }^{13} \mathrm{C}-\mathrm{NMR}$ $\left(\mathrm{C}_{6} \mathrm{D}_{6}, 100.6 \mathrm{MHz}\right): 27.96,28.00,29.19,33.82,39.85,39.92,45.00,62.26,66.89,66.91,69.26,71.80$, 71.87, 72.75, 72.89, 79.85, 79.94, 80.09, 80.52, 100.95, 127.34-129.91 (Car), 136.00, 137.61, 168.89, 169.08, 169.99, 171.88, 172.46, 174.05 ppm; M.S. (MALDI): $923.68[\mathrm{M}+\mathrm{K}]^{+}, 907.70[\mathrm{M}+\mathrm{Na}]^{+}$

8d:(yield: $32 \mathrm{mg}, 40 \%)[\alpha]^{25} \mathrm{D}=-5.3 \mathrm{c}=0.4 \mathrm{CHCl}_{3}$; ${ }^{1} \mathrm{H}-\mathrm{NMR}\left(\mathrm{C}_{6} \mathrm{D}_{6}, 400 \mathrm{MHz}\right): 1.15-1.25\left(\mathrm{~m}, 1 \mathrm{H}, \mathrm{H}_{6 \mathrm{ax}}\right)$, 1.40 (s, 9H, $\left.\left(\mathrm{CH}_{3}\right)_{3} \mathrm{C}-\right), 1.55$ (s, 9H, $\left.\left(\mathrm{CH}_{3}\right)_{3} \mathrm{C}-\right), 1.60$ (s, H3, $\left.\mathrm{CH}_{3} \mathrm{CO}-\right), 1.77$ (s, H3, $\left.\mathrm{CH}_{3} \mathrm{CO}-\right), 1.82(\mathrm{~s}$, $\left.\mathrm{H} 3, \mathrm{CH}_{3} \mathrm{CO}-\right), 2.10$ (s, H3, $\left.\mathrm{CH}_{3} \mathrm{CO}-\right), 2.20$ (ddd, $\left.1 \mathrm{H}, \mathrm{H}_{3 \mathrm{ax}}, \mathrm{J}_{\mathrm{gem}}=\mathrm{J}_{3 \mathrm{ax}-4}=\mathrm{J}_{3 \mathrm{ax}-2}=12 \mathrm{~Hz}\right), 2.35\left(\mathrm{dt}, 1 \mathrm{H}, \mathrm{H}_{6 \mathrm{eq}}\right.$, $\mathrm{J}_{\mathrm{gem}}=10 \mathrm{~Hz}$ and $\left.\mathrm{J}_{6 \mathrm{eq}-5}=\mathrm{J}_{6 \mathrm{eq}-1}=4 \mathrm{~Hz}\right), 2.50\left(\mathrm{dt}, 1 \mathrm{H}, \mathrm{H}_{3 \mathrm{eq}}, \mathrm{J}_{\mathrm{gem}}=10 \mathrm{~Hz}\right.$ and $\left.\mathrm{J}_{3 \mathrm{eq}-2}=\mathrm{J}_{3 \mathrm{eq}-4}=4 \mathrm{~Hz}\right), 2.75(\mathrm{td}, 1 \mathrm{H}$, $\mathrm{H}_{2}, \mathrm{~J}_{2-3 \mathrm{ax}}=\mathrm{J}_{2-1}=12 \mathrm{~Hz}$ and $\left.\mathrm{J}_{2-3 \mathrm{eq}}=4 \mathrm{~Hz}\right), 3.0-3.15\left(\mathrm{~m}, 3 \mathrm{H}, \mathrm{H}_{4}\right.$ and $\left.\mathrm{CH}_{2} \mathrm{Ph}\right), 3.30\left(\mathrm{td}, 1 \mathrm{H}, \mathrm{H}_{1}, \mathrm{~J}_{1-16 \mathrm{ax}}=\mathrm{J}_{1-2}=\right.$ $12 \mathrm{~Hz}$ and $\left.\mathrm{J}_{1-6 \mathrm{eq}}=4 \mathrm{~Hz}\right), 3.40-3.47\left(\mathrm{~m}, 1 \mathrm{H}, \mathrm{GalH}_{5}\right), 3.95\left(\mathrm{bs}, 1 \mathrm{H}, \mathrm{H}_{5}\right), 4.20-4.35\left(\mathrm{~m}, 3 \mathrm{H}, \mathrm{H}_{\mathrm{L}}\right.$ and $2 \mathrm{x}$ $\left.\mathrm{GalH}_{6}\right), 4.45\left(\mathrm{~d}, 1 \mathrm{H}, \mathrm{GalH}_{1}, \mathrm{~J}_{\mathrm{GH} 1-\mathrm{GH} 2}=8 \mathrm{~Hz}\right), 5.15\left(\mathrm{~d}, 1 \mathrm{H}, \mathrm{OCH} \mathrm{HPh}(\mathrm{Bn}), \mathrm{J}_{\mathrm{gem}}=12 \mathrm{~Hz}\right), 5.25(\mathrm{~d}, 1 \mathrm{H}$, $\left.\mathrm{OCH} H \mathrm{Ph}(\mathrm{Bn}), \mathrm{J}_{\mathrm{gem}}=12 \mathrm{~Hz}\right), 5.25\left(\mathrm{dd}, 1 \mathrm{H}, \mathrm{GalH}_{3}, \mathrm{~J}_{\mathrm{GH}-\mathrm{GH} 2}=11 \mathrm{~Hz}\right.$ and $\left.\mathrm{J}_{\mathrm{GH} 3-\mathrm{GH} 4}=3.6 \mathrm{~Hz}\right), 5.60(\mathrm{~d}, 1 \mathrm{H}$, $\left.\mathrm{GalH}_{4}, \mathrm{~J}_{\mathrm{GH} 4-\mathrm{GH} 3}=3.6 \mathrm{~Hz}\right), 5.70\left(\mathrm{dd}, 1 \mathrm{H}, \mathrm{GalH}_{2}, \mathrm{~J}_{\mathrm{GH} 2-\mathrm{GH} 3}=11 \mathrm{~Hz}\right.$ and $\left.\mathrm{J}_{\mathrm{GH} 2-\mathrm{GH} 1}=8 \mathrm{~Hz}\right), 7.20-7.50(\mathrm{~m}, 10 \mathrm{H}$, $\left.\mathrm{H}_{\mathrm{ar}}\right) \mathrm{ppm} ;{ }^{13} \mathrm{C}-\mathrm{NMR}\left(\mathrm{C}_{6} \mathrm{D}_{6}, 75.5 \mathrm{MHz}\right): 19.57,19.95,20.03,20.61,27.69,27.78,28.99,39.61,39.69$, $44.87,61.24,66.73,67.11,69.14,70.33,70.85,79.58,79.60,79.82,80.55,101.16,108.09,127.16-$ 129.69 (Car), 137.39, 168.97, 169.73, 170.00, 171.67, 172.22, 173.2 ppm; M.S. (MALDI): 924.40 $[\mathrm{M}+\mathrm{Na}]^{+}, 908.35[\mathrm{M}+\mathrm{K}]^{+}$

General procedure for the synthesis of compound $(1 S, 2 S, 4 R, 5 S)-4-((R)-1-B e n z y l o x y c a r b o n y l-2-$ phenyl-ethoxy)-5-(2-acetoamido-3,4,6- $O$-triacetyl-2-desoxy- $\beta$-D-glucopyranosyl)-cyclohexane-1,2-

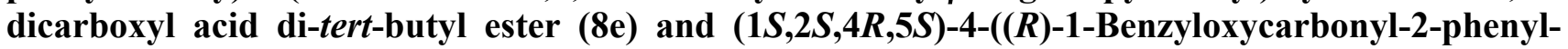
ethoxy)-5-(2-acetoamido-4,6- $O$-dipivaloyl-3- $O$-acetyl-2-desoxy- $\beta$-D-galactopyranosyl)cyclohexane-1,2-dicarboxyl acid di-tert-butyl ester $(\mathbf{8 f})$
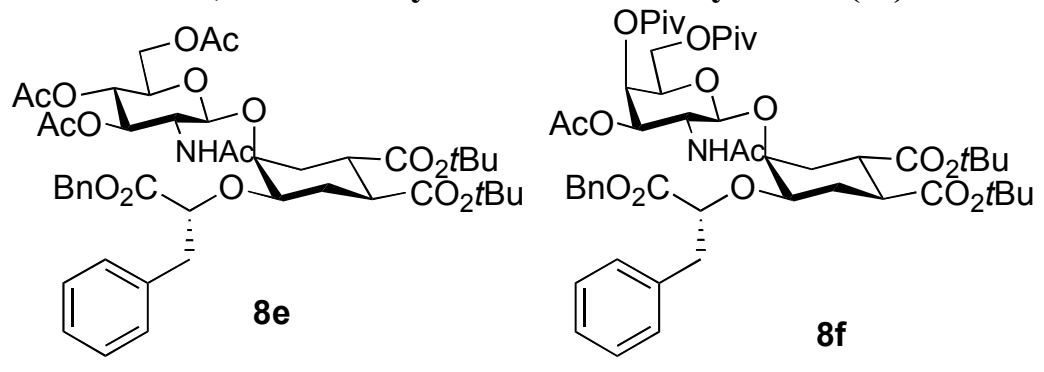

To a solution of monoether $\mathbf{5 b}(50 \mathrm{mg}, 0.09 \mathrm{mmol}, 1 \mathrm{eq})$ and 2-acetoamido-3,4,6- $O$-triacetyl-2desoxy-glucopyranosyl trichloroacetimidate (7e) $(93 \mathrm{mg}, 0.18 \mathrm{mmol}, 2 \mathrm{eq})$ or 2-acetoamido-4,6-Odipivaloyl-3- $O$-acetyl-2-desoxy-galactopyranosyl trichloroacetimidate (7f) $(23 \mathrm{mg}, 0.045 \mathrm{mmol}, 0.5 \mathrm{eq})$ in dry $\mathrm{CH}_{2} \mathrm{Cl}_{2}(1.5 \mathrm{~mL})$, under $\mathrm{N}_{2}$ and in presence of molecular sieves $4 \AA$ acid washed $(150 \mathrm{mg})$ was stirred for $10 \mathrm{~min}$ at R.T., after this time TfOH $(1.7 \mathrm{mg}, 0.009 \mathrm{mmol}, 0.1 \mathrm{eq})$ was added in it. The reaction mixture was stirred at R.T. for $3 \mathrm{~h}$ monitoring by TLC (petroleum ether/AcOEt: 75:25), after this time other 0.1 eq of $\mathrm{TfOH}$ was added and the reaction was heated at $40^{\circ} \mathrm{C}$ overnight. After reaction completion, TEA was added until $\mathrm{pH}=7$ was reached and the solvent was evaporated under reduced pressure. The crude product was purified by flash chromatography on silica gel (petroleum ether/AcOEt 1:1) obtaining the product as amorphous white solid.

8e:(yield: $7 \mathrm{mg}, 18 \%)[\alpha]^{25}=1.5 \mathrm{c}=0.2 \mathrm{CHCl}_{3} ;{ }^{1} \mathrm{H}-\mathrm{NMR}\left(\mathrm{C}_{6} \mathrm{D}_{6}, 400 \mathrm{MHz}\right): 1.20-1.30\left(\mathrm{~m}, 1 \mathrm{H}, \mathrm{H}_{6 \mathrm{ax}}\right)$, $1.40\left(\mathrm{~s}, 9 \mathrm{H},\left(\mathrm{CH}_{3}\right)_{3} \mathrm{C}-\right), 1.50\left(\mathrm{~s}, 9 \mathrm{H},\left(\mathrm{CH}_{3}\right)_{3} \mathrm{C}-\right), 1.75-1.80\left(\mathrm{~m}, 1 \mathrm{H}, \mathrm{H}_{3 \mathrm{ax}}\right), 1.80\left(\mathrm{~s}, \mathrm{H} 3, \mathrm{CH}_{3} \mathrm{CO}-\right), 1.85$ (s, $\left.\mathrm{H} 3, \mathrm{CH}_{3} \mathrm{CO}-\right), 1.90$ (s, H3, $\left.\mathrm{CH}_{3} \mathrm{CO}-\right), 2.05$ (s, H3, $\left.\mathrm{CH}_{3} \mathrm{CONH}-\right), 2.15-2.25$ (m,1H, $\mathrm{H}_{6 \mathrm{eq}}$ ), 2.38 (dt, $1 \mathrm{H}$, $\mathrm{H}_{3 \text { eq }}, \mathrm{J}_{\mathrm{gem}}=10 \mathrm{~Hz}$ and $\mathrm{J}_{3 \mathrm{eq}-2}=\mathrm{J}_{3 \mathrm{eq}-4}=4 \mathrm{~Hz}$ ), $2.70\left(\mathrm{td}, 1 \mathrm{H}, \mathrm{H}_{2}, \mathrm{~J}_{2-3 \mathrm{ax}}=\mathrm{J}_{2-1}=12 \mathrm{~Hz}\right.$ and $\left.\mathrm{J}_{2-3 \mathrm{eq}}=4 \mathrm{~Hz}\right), 2.90-$ $3.05\left(\mathrm{~m}, 3 \mathrm{H}, \mathrm{H}_{4}\right.$ and $\left.\mathrm{CH}_{2} \mathrm{Ph}\right), 3.30\left(\mathrm{td}, 1 \mathrm{H}, \mathrm{H}_{1}, \mathrm{~J}_{1-6 \mathrm{ax}}=\mathrm{J}_{1-2}=12 \mathrm{~Hz}\right.$ and $\left.\mathrm{J}_{1-6 \mathrm{eq}}=4 \mathrm{~Hz}\right), 3.50-3.60(\mathrm{~m}, 1 \mathrm{H}$, 
$\left.\mathrm{GlcNAcH}_{5}\right), 3.90\left(\mathrm{bs}, 1 \mathrm{H}, \mathrm{H}_{5}\right), 4.00\left(\mathrm{t}, 1 \mathrm{H}, \mathrm{HL}, \mathrm{J}_{\mathrm{HL}-\mathrm{CH} 2 \mathrm{Ph}}=6 \mathrm{~Hz}\right), 4.25-4.35\left(\mathrm{~m}, 2 \mathrm{H}, \mathrm{GlcNAcH}_{6}\right.$ and $\left.\mathrm{GlcNAcH}_{2}\right), 4.45\left(\mathrm{dd}, 1 \mathrm{H}, \mathrm{GlcNAcH}_{6}, \mathrm{~J}_{\mathrm{gem}}=12 \mathrm{~Hz}\right.$ and $\left.\mathrm{J}_{\mathrm{GH} 6-\mathrm{GH} 5}=5 \mathrm{~Hz}\right), 4.83\left(\mathrm{~d}, 1 \mathrm{H}, \mathrm{GlcNAcH}_{1}, \mathrm{~J}_{\mathrm{GH} 1-}\right.$ $\left.\mathrm{GH}_{2}=8.6 \mathrm{~Hz}\right), 5.02\left(\mathrm{~s}, 2 \mathrm{H}, \mathrm{OCH}_{2} \mathrm{Ph}(\mathrm{Bn})\right), 5.35\left(\mathrm{t}, 1 \mathrm{H}, \mathrm{GlcNHAcH}_{4}, \mathrm{~J}_{\mathrm{GH} 4-\mathrm{GH} 2}=\mathrm{J}_{\mathrm{GH} 4-\mathrm{GH} 5}=10 \mathrm{~Hz}\right), 5.42(\mathrm{t}$, $\left.1 \mathrm{H}, \mathrm{GlcNAcH}_{3}, \mathrm{~J}_{\mathrm{GH} 3-\mathrm{GH} 2}=\mathrm{J}_{\mathrm{GH} 3-\mathrm{GH} 4}=10 \mathrm{~Hz}\right), 6.03\left(\mathrm{~d}, 1 \mathrm{H}, \mathrm{NH}, \mathrm{J}_{\mathrm{NH}-\mathrm{GH} 2}=10 \mathrm{~Hz}\right), 7.20-7.50\left(\mathrm{~m}, 10 \mathrm{H}, \mathrm{H}_{\mathrm{ar}}\right)$ ppm. ${ }^{13} \mathrm{C}-\mathrm{NMR}\left(\mathrm{C}_{6} \mathrm{D}_{6}, 50.3 \mathrm{MHz}\right): 20.19,20.29,20.40,23.14,28.42,30.12,33.53,39.21,39.89,44.77$, 54.54, 62.52, 67.11, 69.42, 73.25, 77.91, 79.54, 79.59, 80.60, 81.54, 101.28, 127.47-129.64 (Car), $130.12,136.89,169.06,170.30,172.31,172.42,173.94$ ppm. MS (ESI): $924.5\left[\mathrm{MH}_{2}+\mathrm{K}\right]^{3+}, 923.5$ $[\mathrm{MH}+\mathrm{K}]^{2+}, 922.5[\mathrm{M}+\mathrm{K}]^{+}, 907.5[\mathrm{MH}+\mathrm{Na}]^{2+}, 906.5[\mathrm{M}+\mathrm{Na}]^{+}$

8f: (yield: $7 \mathrm{mg}, 27 \%)[\alpha]^{25}{ }_{\mathrm{D}}=-15.3 \mathrm{c}=1 \mathrm{CHCl}_{3} ;{ }^{1} \mathrm{H}-\mathrm{NMR}\left(\mathrm{C}_{6} \mathrm{D}_{6}, 400 \mathrm{MHz}\right): 1.20-1.40\left(\mathrm{~m}, 2 \mathrm{H}, \mathrm{H}_{6 \mathrm{x}}\right.$ and $\left.\mathrm{H}_{3 \mathrm{ax}}\right), 1.20$ (s, 9H, $\left(\mathrm{CH}_{3}\right)_{3} \mathrm{C}$ - (Piv)), 1.25 (s, 9H, $\left.\left(\mathrm{CH}_{3}\right)_{3} \mathrm{C}-(\mathrm{Piv})\right), 1.35\left(\mathrm{~s}, 9 \mathrm{H},\left(\mathrm{CH}_{3}\right)_{3} \mathrm{C}-(t\right.$ - $\left.\mathrm{Bu})\right), 1.47(\mathrm{~s}$, $\left.9 \mathrm{H},\left(\mathrm{CH}_{3}\right)_{3} \mathrm{C}-(t-\mathrm{Bu})\right), 1.83\left(\mathrm{~s}, 3 \mathrm{H}, \mathrm{CH}_{3} \mathrm{CO}-\right), 2.05-2.10\left(\mathrm{~m}, 1 \mathrm{H}, \mathrm{H}_{3 \mathrm{eq}}\right), 2.07\left(\mathrm{~s}, 3 \mathrm{H}, \mathrm{CH}_{3} \mathrm{CONH}\right), 2.40$ $\left(\mathrm{dt}, 1 \mathrm{H}, \mathrm{H}_{6 \mathrm{eq}}, \mathrm{J}_{\mathrm{gem}}=12 \mathrm{~Hz}\right.$ and $\left.\mathrm{J}_{3 \mathrm{eq}-2}=\mathrm{J}_{3 \mathrm{eq}-4}=4 \mathrm{~Hz}\right), 2.60-2.70\left(\mathrm{~m}, 1 \mathrm{H}, \mathrm{H}_{2}\right), 2.80-3.00\left(\mathrm{~m}, 3 \mathrm{H}, \mathrm{H}_{4}\right.$ and $\left.\mathrm{CH}_{2} \mathrm{Ph}\right), 3.30\left(\mathrm{td}, 1 \mathrm{H}, \mathrm{H}_{1}, \mathrm{~J}_{1-6 \mathrm{ax}}=\mathrm{J}_{1-2}=12 \mathrm{~Hz}\right.$ and $\left.\mathrm{J}_{1-6 \mathrm{eq}}=4 \mathrm{~Hz}\right), 3.75-3.83\left(\mathrm{~m}, 1 \mathrm{H}, \mathrm{GalNAcH}_{5}\right), 3.90(\mathrm{t}$, $\left.1 \mathrm{H}, \mathrm{H}_{\mathrm{L}}, \mathrm{J}_{\mathrm{HL}-\mathrm{CH} 2 \mathrm{Ph}}=6 \mathrm{~Hz}\right), 3.97\left(\mathrm{bs}, 1 \mathrm{H}, \mathrm{H}_{5}\right), 4.25\left(\mathrm{dd}, 1 \mathrm{H}, \mathrm{GalNAcH}_{6}, \mathrm{~J}_{\mathrm{gem}}=11 \mathrm{~Hz}\right.$ and $\left.\mathrm{J}_{\mathrm{GH} 6-\mathrm{GH} 5}=8 \mathrm{~Hz}\right)$, $4.48\left(\mathrm{dd}, 2 \mathrm{H}, \mathrm{GalNAcH}_{6}, \mathrm{~J}_{\mathrm{gem}}=11 \mathrm{~Hz}\right.$ and $\left.\mathrm{J}_{\mathrm{GH}-\mathrm{GH} 5}=6 \mathrm{~Hz}\right), 4.75-4.85\left(\mathrm{~m}, 2 \mathrm{H}, \mathrm{GalNAcH}_{1}\right.$ and GalNAcH $\left._{2}\right), 4.95\left(\mathrm{~s}, 2 \mathrm{H}, \mathrm{OCH}_{2} \mathrm{Ph}(\mathrm{Bn})\right)$, 4.97-5.04 (m, 1H, GalNAcH 3$), 5.62\left(\mathrm{~d}, 1 \mathrm{H}, \mathrm{GalNAcH}_{4}, \mathrm{~J}_{\mathrm{GH} 4-}\right.$ $\left.\mathrm{GH}_{3}=\mathrm{J}_{\mathrm{GH} 4-\mathrm{GH} 5}=3.5 \mathrm{~Hz}\right), 6.40\left(\mathrm{~d}, 1 \mathrm{H}, \mathrm{NH}, \mathrm{J}_{\mathrm{NH}-\mathrm{GH} 2}=10 \mathrm{~Hz}\right), 7.20-7.50\left(\mathrm{~m}, 10 \mathrm{H}, \mathrm{H}_{\mathrm{ar}}\right) \mathrm{ppm} ;{ }^{13} \mathrm{C}-\mathrm{NMR}-$ HETCOR $\left(\mathrm{C}_{6} \mathrm{D}_{6}, 400 \mathrm{MHz}\right): 20.59,23.52,27.41,27.46,28.05,28.21,28.42,33.70,39.14,39.94,45.00$, $50.65,61.54,66.77,67.44,71.14,72.47,77.35,80.99,102.16,127.67,128.14,129.11,129.80 \mathrm{ppm}$. MS (F.A.B $\left.{ }^{+}\right): 968[\mathrm{M}+\mathrm{H}]^{+}, 967[\mathrm{M}]$

\section{General procedure for the synthesis of compounds 9c-9f}
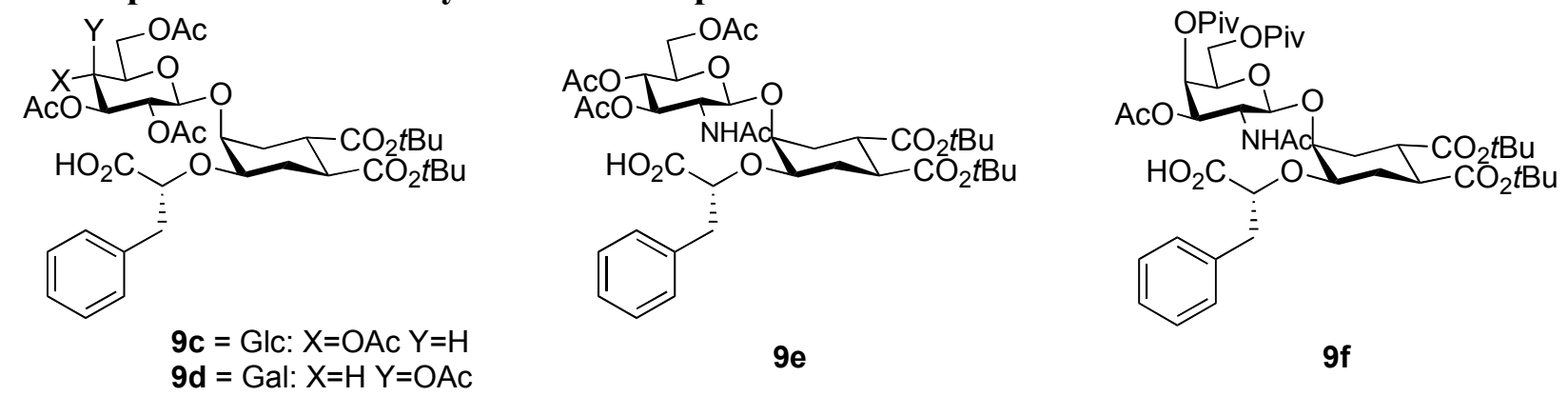

To a solution of compound $8 \mathbf{c}-8 \mathbf{8}(1 \mathrm{eq})$ in $\mathrm{MeOH} / \mathrm{H}_{2} \mathrm{O} 90: 10(0.5 \mathrm{M})$ a catalytic amount of $\mathrm{Pd}-\mathrm{C} 10 \%$ was added. The mixture was stirred under $\mathrm{H}_{2}$ atmosphere at room temperature, monitoring by TLC $\left(\mathrm{CHCl}_{3} / \mathrm{MeOH}\right.$ : 90:10). After reaction completion, the mixture was filtered through a celite pad, washing several times with methanol. The collected organic phase was evaporated under reduced pressure and the crude was purified by flash chromatography on silica gel. The products 9c-9f were obtained as amorphous white solid.

\section{$(1 S, 2 S, 4 R, 5 S)-4-((R)-1-C a r b o x y l-2-p h e n y l-e t h o x y)-5-(2,3,4,6$-tetra- $O$-acetyl- $\beta$-D-glucopyranosyl)-} cyclohexane-1,2-dicarboxyl acid di-tert-butyl ester (9c):

Chromatography eluent: $\left(\mathrm{CHCl}_{3} / \mathrm{MeOH}\right.$ : 95:5), (yield: $\left.15 \mathrm{mg}, 70 \%\right) ;{ }^{1} \mathrm{H}-\mathrm{NMR}\left(\mathrm{CD}_{3} \mathrm{OD}, 300 \mathrm{MHz}\right)$ : 1.25-1.35 (m, $\left.1 \mathrm{H}, \mathrm{H}_{6 \mathrm{ax}}\right), 1.40\left(\mathrm{~s}, 9 \mathrm{H},\left(\mathrm{CH}_{3}\right)_{3} \mathrm{C}-\right), 1.45\left(\mathrm{~s}, 9 \mathrm{H},\left(\mathrm{CH}_{3}\right)_{3} \mathrm{C}-\right), 1.50-1.70\left(\mathrm{~m}, 1 \mathrm{H}, \mathrm{H}_{3 \mathrm{ax}}\right), 1.95(\mathrm{~s}$, $3 \mathrm{H}, \mathrm{CH}_{3} \mathrm{CO}-$ ), 1.95-2.03 (m, 1H, $\mathrm{H}_{3 \mathrm{eq}}$ ), 2.03 (s, 3H, $\left.\mathrm{CH}_{3} \mathrm{CO}-\right), 2.07$ (s, 6H, 2 x CH $3 \mathrm{CO}_{3}$ ), 2.12-2.20 (m, $\left.1 \mathrm{H}, \mathrm{H}_{6 \mathrm{eq}}\right), 2.4\left(\mathrm{td}, 1 \mathrm{H}, \mathrm{H}_{2}, \mathrm{~J}_{2-3 \mathrm{ax}}=\mathrm{J}_{2-1}=13\right.$ and $\left.\mathrm{Hz} \mathrm{J}_{2-3 \mathrm{eq}}=4 \mathrm{~Hz}\right), 2.65\left(\mathrm{td}, 1 \mathrm{H}, \mathrm{H}_{1}, \mathrm{~J}_{1-6 \mathrm{ax}}=\mathrm{J}_{1-2}=13 \mathrm{~Hz}\right.$ and $\mathrm{J}_{1-}$ $\left.{ }_{6 e q}=4 \mathrm{~Hz}\right), 2.9\left(\mathrm{dd}, 1 \mathrm{H}, \mathrm{CHHPh}, \mathrm{J}_{\mathrm{gem}}=14 \mathrm{~Hz}\right.$ and $\left.\mathrm{J}_{\mathrm{CHPh}-\mathrm{HL}}=10 \mathrm{~Hz}\right), 3.05-3.20(\mathrm{~m}, 2 \mathrm{H}, \mathrm{CH} H \mathrm{Ph}$, and $\left.\mathrm{GlcH}_{5}\right), 3.25-3.35\left(\mathrm{~m}, 1 \mathrm{H}, \mathrm{H}_{4}\right), 3.80\left(\mathrm{bs}, 1 \mathrm{H}, \mathrm{H}_{5}\right), 3.85\left(\mathrm{~d}, 1 \mathrm{H}, \mathrm{GlcH}_{1}, \mathrm{~J}_{\mathrm{GH} 1-\mathrm{GH} 2}=8 \mathrm{~Hz}\right), 4.05(\mathrm{dd}, 1 \mathrm{H}$, $\mathrm{GlcH}_{6}, \mathrm{~J}_{\mathrm{gem}}=13 \mathrm{~Hz}$ and $\left.\mathrm{J}_{\mathrm{GH} 6-\mathrm{GH} 5}=2 \mathrm{~Hz}\right), 4.17\left(\mathrm{dd}, 1 \mathrm{H}, \mathrm{GlcH}_{6}, \mathrm{~J}_{\mathrm{gem}}=13 \mathrm{~Hz}\right.$ and $\left.\mathrm{J}_{\mathrm{GH} 6-\mathrm{GH} 5}=4 \mathrm{~Hz}\right), 4.30$ $\left(\mathrm{dd}, 1 \mathrm{H}, \mathrm{H}_{\mathrm{L}}, \mathrm{J}_{\mathrm{HL}-\mathrm{CH} 2 \mathrm{Ph}}=10\right.$ and $\left.3 \mathrm{~Hz}\right), 4.75\left(\mathrm{dd}, 1 \mathrm{H}, \mathrm{GlcH}_{4}, \mathrm{~J}_{\mathrm{GH} 4-\mathrm{GH} 3}=10\right.$ and $\left.\mathrm{J}_{\mathrm{GH} 4-\mathrm{GH} 5}=8 \mathrm{~Hz}\right), 4.80-4.90$ $\left(\mathrm{m}, 1 \mathrm{H}, \mathrm{GlcH}_{2}\right), 5.10\left(\mathrm{t}, 1 \mathrm{H}, \mathrm{GlcH}_{3}, \mathrm{~J}_{\mathrm{GH} 3-\mathrm{GH} 2}=\mathrm{J}_{\mathrm{GH} 3-\mathrm{GH} 4}=10 \mathrm{~Hz}\right), 7.25-7.45\left(\mathrm{~m}, 5 \mathrm{H}, \mathrm{H}_{\mathrm{ar}}\right)$ ppm; MS (MALDI): $817.93[\mathrm{M}+\mathrm{Na}]^{+}, 839.98[\mathrm{M}+\mathrm{K}]^{+}$ 
$(1 S, 2 S, 4 R, 5 S)-4-((R)-1-C a r b o x y l-2-p h e n y l-e t h o x y)-5-(2,3,4,6$-tetra- $O$-acetyl- $\beta$-D-

galactopyranosyl)-cyclohexane-1,2-dicarboxyl acid di-tert-butyl ester (9d):

Chromatography eluent: $\left(\mathrm{CHCl}_{3} / \mathrm{MeOH}\right.$ : 95:5), (yield: $\left.23 \mathrm{mg}, 93 \%\right) ;{ }^{1} \mathrm{H}-\mathrm{NMR}\left(\mathrm{CD}_{3} \mathrm{OD}, 400 \mathrm{MHz}\right)$ :

1.30-1.40 (m, 1H, $\left.\mathrm{H}_{6 \mathrm{ax}}\right), 1.43\left(\mathrm{~s}, 1 \mathrm{H},\left(\mathrm{CH}_{3}\right)_{3} \mathrm{C}-\right), 1.45\left(\mathrm{~s}, 1 \mathrm{H},\left(\mathrm{CH}_{3}\right)_{3} \mathrm{C}-\right), 1.65$ (ddd, $1 \mathrm{H}, \mathrm{H}_{3 \mathrm{ax}}, \mathrm{J}_{\text {gem }}=\mathrm{J}_{3 \mathrm{ax}-}$ $\left.{ }_{4}=\mathrm{J}_{3 \mathrm{ax}-2}=12 \mathrm{~Hz}\right), 1.98\left(\mathrm{~s}, \mathrm{H} 3, \mathrm{CH}_{3} \mathrm{CO}-\right), 2.02-2.20\left(\mathrm{~m}, 2 \mathrm{H}, \mathrm{H}_{6 \mathrm{eq}}\right.$ and $\left.\mathrm{H}_{3 \mathrm{eq}}\right), 2.08\left(\mathrm{~s}, 3 \mathrm{H}, \mathrm{CH}_{3} \mathrm{CO}-\right), 2.10(\mathrm{~s}$, $\left.3 \mathrm{H}, \mathrm{CH}_{3} \mathrm{CO}-\right), 2.18\left(\mathrm{~s}, 3 \mathrm{H}, \mathrm{CH}_{3} \mathrm{CO}-\right), 2.43\left(\mathrm{td}, 1 \mathrm{H}, \mathrm{H}_{2}, \mathrm{~J}_{2-3 \mathrm{ax}}=\mathrm{J}_{2-1}=13 \mathrm{~Hz}\right.$ and $\left.\mathrm{J}_{2-3 \mathrm{eq}}=4 \mathrm{~Hz}\right), 2.70(\mathrm{td}, 1 \mathrm{H}$, $\mathrm{H}_{1}, \mathrm{~J}_{1-6 \mathrm{ax}}=\mathrm{J}_{1-2}=13$ and $\left.\mathrm{Hz}_{\mathrm{I}-6 \mathrm{eq}}=4 \mathrm{~Hz}\right), 2.94\left(\mathrm{dd}, 1 \mathrm{H}, \mathrm{CHHPh}, \mathrm{J}_{\mathrm{gem}}=14 \mathrm{~Hz}\right.$ and $\left.\mathrm{J}_{\mathrm{CHPh}-\mathrm{HL}}=10 \mathrm{~Hz}\right), 3.17$ (dd, $1 \mathrm{H}, \mathrm{CH} H \mathrm{Ph}, \mathrm{J}_{\mathrm{gem}}=14 \mathrm{~Hz}$ and $\left.\mathrm{J}_{\mathrm{CHPh}-\mathrm{HL}}=3.4 \mathrm{~Hz}\right), 3.28-3.35\left(\mathrm{~m}, 1 \mathrm{H}, \mathrm{H}_{4}\right), 3.47-3.52\left(\mathrm{~m}, 1 \mathrm{H}, \mathrm{GalH}_{5}\right)$, $3.83\left(\mathrm{bs}, 1 \mathrm{H}, \mathrm{H}_{5}\right), 4.00\left(\mathrm{~d}, 1 \mathrm{H}, \mathrm{GalH}_{1}, \mathrm{~J}_{\mathrm{GH} 1-\mathrm{GH} 2}=8 \mathrm{~Hz}\right), 4.05-4.13\left(\mathrm{~m}, 2 \mathrm{H}, 2 \times \mathrm{GalH}_{6}\right), 4.40\left(\mathrm{dd}, 1 \mathrm{H}, \mathrm{H}_{\mathrm{L}}\right.$, $\mathrm{J}_{\mathrm{HL}-\mathrm{CH} 2 \mathrm{Ph}}=10$ and $\left.3.4 \mathrm{~Hz}\right), 4.75\left(\mathrm{dd}, 1 \mathrm{H}, \mathrm{GalH}_{3}, \mathrm{~J}_{\mathrm{GH} 3-\mathrm{GH} 2}=10 \mathrm{~Hz}\right.$ and $\left.\mathrm{J}_{\mathrm{GH} 3-\mathrm{GH} 4}=3 \mathrm{~Hz}\right), 4.95(\mathrm{dd}, 1 \mathrm{H}$, $\mathrm{GalH}_{2}, \mathrm{~J}_{\mathrm{GH} 2-\mathrm{GH} 3}=10 \mathrm{~Hz}$ and $\left.\mathrm{J}_{\mathrm{GH} 2-\mathrm{GH} 1}=8 \mathrm{~Hz}\right), 5.25-5.30\left(\mathrm{~m}, 1 \mathrm{H}, \mathrm{GalH}_{4}\right), 7.30-7.45\left(\mathrm{~m}, 5 \mathrm{H}, \mathrm{H}_{\mathrm{ar}}\right)$.ppm; MS (MALDI): $856.40[\mathrm{M}+\mathrm{Na}+\mathrm{K}]^{2+}, 840.44[\mathrm{M}+2 \mathrm{Na}]^{2+}, 834.42[\mathrm{M}+\mathrm{K}]^{+}, 818.44[\mathrm{M}+\mathrm{Na}]^{+}$

\section{$(1 S, 2 S, 4 R, 5 S)-4-((R)-1-C a r b o x y l-2-p h e n y l-e t h o x y)-5-(2-a c e t o a m i d o-3,4,6-O$-triacetyl-2-desoxy- $\beta$ - D-glucopyranosyl)-cyclohexane-1,2-dicarboxyl acid di-tert-butyl ester (9e):}

Chromatography eluent: $\left(\mathrm{CHCl}_{3} / \mathrm{MeOH}\right.$ : 98:2), (yield: $\left.8 \mathrm{mg}, 84 \%\right) ;{ }^{1} \mathrm{H}-\mathrm{NMR}\left(\mathrm{CD}_{3} \mathrm{OD}, 300 \mathrm{MHz}\right): 1.20-$ $1.30\left(\mathrm{~m}, 1 \mathrm{H}, \mathrm{H}_{6 \mathrm{ax}}\right), 1.39\left(\mathrm{~s}, 9 \mathrm{H},\left(\mathrm{CH}_{3}\right)_{3} \mathrm{C}-\right), 1.42\left(\mathrm{~s}, 9 \mathrm{H},\left(\mathrm{CH}_{3}\right)_{3} \mathrm{C}-\right), 1.60\left(\mathrm{ddd}, 1 \mathrm{H}, \mathrm{H}_{3 \mathrm{ax}}, \mathrm{J}_{\mathrm{gem}}=\mathrm{J}_{3 \mathrm{ax}-2}=\mathrm{J}_{3 \mathrm{ax}-}\right.$ $4=12 \mathrm{~Hz}), 1.94\left(\mathrm{~s}, 3 \mathrm{H}, \mathrm{CH}_{3} \mathrm{CO}-\right), 1.98\left(\mathrm{~s}, 3 \mathrm{H}, \mathrm{CH}_{3} \mathrm{CO}-\right), 2.03$ (s, 3H, $\left.\mathrm{CH}_{3} \mathrm{CO}-\right), 2.07$ (s, 3H, $\mathrm{CH}_{3} \mathrm{CONH}-$ ), 2.00-2.10 (m, $2 \mathrm{H}, \mathrm{H}_{3 \mathrm{eq}}$ and $\left.\mathrm{H}_{6 \mathrm{eq}}\right), 2.35\left(\mathrm{td}, 1 \mathrm{H}, \mathrm{H}_{2}, \mathrm{~J}_{2-3 \mathrm{ax}}=\mathrm{J}_{2-1}=12 \mathrm{~Hz}\right.$ and $\left.\mathrm{J}_{2-3 \mathrm{eq}}=4 \mathrm{~Hz}\right), 2.65(\mathrm{td}, 1 \mathrm{H}$, $\mathrm{H}_{1}, \mathrm{~J}_{1-6 \mathrm{ax}}=\mathrm{J}_{1-2}=13 \mathrm{~Hz}$ and $\left.\mathrm{J}_{1-6 \mathrm{eq}}=4 \mathrm{~Hz}\right), 3.05\left(\mathrm{dd}, 1 \mathrm{H}, \mathrm{CHHPh}, \mathrm{J}_{\text {gem }}=14 \mathrm{~Hz}\right.$ and $\left.\mathrm{J}_{\mathrm{CHPh}-\mathrm{HL}}=8 \mathrm{~Hz}\right), 3.15$ $\left(\mathrm{dd}, 1 \mathrm{H}, \mathrm{CH} H \mathrm{Ph}, \mathrm{J}_{\mathrm{gem}}=14 \mathrm{~Hz}\right.$ and $\left.\mathrm{J}_{\mathrm{CHPh}-\mathrm{HL}}=4 \mathrm{~Hz}\right), 3.25-3.40\left(\mathrm{~m}, 2 \mathrm{H}, \mathrm{H}_{4}\right.$ and $\left.\mathrm{GlcNAcH}_{5}\right), 3.85-3.95$ $\left(\mathrm{m}, 2 \mathrm{H}, \mathrm{H}_{\mathrm{L}}\right.$ and $\left.\mathrm{H}_{5}\right), 4.05\left(\mathrm{dd}, 1 \mathrm{H}, \mathrm{GlcNAcH}_{6}, \mathrm{~J}_{\mathrm{gem}}=13 \mathrm{~Hz}\right.$ and $\left.\mathrm{J}_{\mathrm{GH} 6-\mathrm{GH} 5}=2 \mathrm{~Hz}\right), 4.17-4.30(\mathrm{~m}, 2 \mathrm{H}$, $\mathrm{GlcNAcH}_{6}$ and $\left.\mathrm{GlcNAcH}_{2}\right), 4.70\left(\mathrm{~d}, 1 \mathrm{H}, \mathrm{GlcNAcH}_{1}, \mathrm{~J}_{\mathrm{GH} 1-\mathrm{GH}}=9 \mathrm{~Hz}\right), 4.90-5.00\left(\mathrm{~m}, 2 \mathrm{H}, \mathrm{GlcNAcH}_{4}\right.$ and $\left.\mathrm{GlcNAcH}_{3}\right), 7.25-7.45\left(\mathrm{~m}, 5 \mathrm{H}, \mathrm{H}_{\mathrm{ar}}\right)$ ppm; MS (ESI): $816.5[\mathrm{M}+\mathrm{Na}]^{+}$

$(1 S, 2 S, 4 R, 5 S)-4-((R)-1-C a r b o x y l-2-p h e n y l-e t h o x y)-5-(2-a c e t o a m i d o-4,6-O$-dipivaloyl-3-O-acetyl-2desoxy- $\beta$-galactopyranosyl)-cyclohexane-1,2-dicarboxyl acid di-tert-butyl ester (9f): Used without further purification, (yield: $17 \mathrm{mg}, 97 \%)$; ${ }^{1} \mathrm{H}-\mathrm{NMR}\left(\mathrm{CD}_{3} \mathrm{OD}, 300 \mathrm{MHz}\right): 1.15-1.20\left(\mathrm{~m}, 1 \mathrm{H}, \mathrm{H}_{6 \mathrm{ax}}\right), 1.20$ $\left(\mathrm{s}, 9 \mathrm{H},\left(\mathrm{CH}_{3}\right)_{3} \mathrm{C}-(\mathrm{Piv})\right), 1.25$ (s, 9H, $\left.\left(\mathrm{CH}_{3}\right)_{3} \mathrm{C}-(\mathrm{Piv})\right), 1.40\left(\mathrm{~s}, 18 \mathrm{H}, 2 \times\left(\mathrm{CH}_{3}\right)_{3} \mathrm{C}-(t \mathrm{Bu})\right), 1.65$ (ddd, $1 \mathrm{H}$, $\left.\mathrm{H}_{3 \mathrm{ax}} \mathrm{J}_{\mathrm{gem}}=\mathrm{J}_{3 \mathrm{ax}-4}=\mathrm{J}_{3 \mathrm{ax}-2}=12 \mathrm{~Hz}\right), 1.87\left(\mathrm{~s}, 3 \mathrm{H}, \mathrm{CH}_{3} \mathrm{CO}-\right), 1.90\left(\mathrm{~s}, 3 \mathrm{H}, \mathrm{CH}_{3} \mathrm{CONH}-\right), 2.00-2.15\left(\mathrm{~m}, 2 \mathrm{H}, \mathrm{H}_{3 \mathrm{eq}}\right.$ and $\left.\mathrm{H}_{6 \mathrm{eq}}\right), 2.40\left(\mathrm{td}, 1 \mathrm{H}, \mathrm{H}_{2}, \mathrm{~J}_{2-3 \mathrm{ax}}=\mathrm{J}_{2-1}=13 \mathrm{~Hz}\right.$ and $\left.\mathrm{Hz} \mathrm{J}_{2-3 \mathrm{eq}}=4 \mathrm{~Hz}\right), 2.70\left(\mathrm{td}, 1 \mathrm{H}, \mathrm{H}_{1}, \mathrm{~J}_{1-6 \mathrm{ax}}=\mathrm{J}_{1-2}=13 \mathrm{~Hz}\right.$ and $\left.\mathrm{J}_{1-6 \mathrm{eq}}=4 \mathrm{~Hz}\right), 3.07\left(\mathrm{dd}, 1 \mathrm{H}, \mathrm{CH} \mathrm{HPh}, \mathrm{J}_{\mathrm{gem}}=14 \mathrm{~Hz}\right.$ and $\left.\mathrm{J}_{\mathrm{CHPh}-\mathrm{HL}}=8 \mathrm{~Hz}\right), 3.15\left(\mathrm{dd}, 1 \mathrm{H}, \mathrm{CH} H \mathrm{Ph}, \mathrm{J}_{\mathrm{gem}}=\right.$ $\left.14 \mathrm{~Hz} \mathrm{~J}_{\mathrm{CHPh}-\mathrm{HL}}=4 \mathrm{~Hz}\right), 3.40-3.48\left(\mathrm{~m}, 1 \mathrm{H}, \mathrm{H}_{4}\right)$ 3.60-3.70 (m, 1H, GalNAcH $\left.)_{5}\right), 3.95\left(\mathrm{bs}, 1 \mathrm{H}, \mathrm{H}_{5}\right), 4.05$ $\left(\mathrm{dd}, 1 \mathrm{H}, \mathrm{GalNAcH}{ }_{6}, \mathrm{~J}_{\mathrm{gem}}=11 \mathrm{~Hz}\right.$ and $\left.\mathrm{J}_{\mathrm{GH} 6-\mathrm{GH}}=8 \mathrm{~Hz}\right), 4.10\left(\mathrm{dd}, 1 \mathrm{H}, \mathrm{GlcNAcH}_{6}, \mathrm{~J}_{\mathrm{gem}}=11 \mathrm{~Hz}\right.$ and $\mathrm{J}_{\mathrm{GH} 6-}$ $\left.\mathrm{GH}_{5}=8 \mathrm{~Hz}\right), 4.20\left(\mathrm{dd}, 1 \mathrm{H}, \mathrm{GlcNAcH}_{2}, \mathrm{~J}_{\mathrm{GH} 2-\mathrm{GH} 3}=11 \mathrm{~Hz}\right.$ and $\left.\mathrm{J}_{\mathrm{GH} 2-\mathrm{GH} 1}=9 \mathrm{~Hz}\right), 4.32-4.40\left(\mathrm{~m}, 1 \mathrm{H}, \mathrm{H}_{\mathrm{L}}\right), 4.60$ $\left(\mathrm{dd}, 1 \mathrm{H}, \mathrm{GalNAcH}{ }_{3}, \mathrm{~J}_{\mathrm{GH} 3-\mathrm{GH} 2}=11 \mathrm{~Hz}\right.$ and $\left.\mathrm{J}_{\mathrm{GH} 3-\mathrm{GH} 4}=3 \mathrm{~Hz}\right), 4.67\left(\mathrm{~d}, 1 \mathrm{H}, \mathrm{GalNAcH}_{1}, \mathrm{~J}_{\mathrm{GH} 1-\mathrm{GH} 2}=9 \mathrm{~Hz}\right)$, $5.23\left(\mathrm{~d}, 1 \mathrm{H}, \mathrm{GalNAcH} 4, \mathrm{~J}_{\mathrm{GH} 4-\mathrm{GH} 3}=\mathrm{J}_{\mathrm{GH} 4-\mathrm{GH} 5}=3 \mathrm{~Hz}\right), 7.25-7.40\left(\mathrm{~m}, 5 \mathrm{H}, \mathrm{H}_{\mathrm{ar}}\right) \mathrm{ppm}$; MS (MALDI): 938.39 $[\mathrm{M}+\mathrm{Na}+\mathrm{K}]^{2+}, 922.39[\mathrm{M}+2 \mathrm{Na}]^{2+}, 916.34[\mathrm{M}+\mathrm{K}]^{+}, 900.34[\mathrm{M}+\mathrm{Na}]^{+}$

General procedure for the synthesis of compounds $2 \mathrm{c}-2 \mathrm{f}$ 


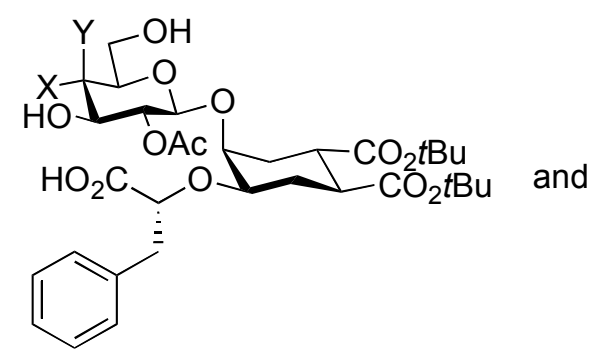

2c $=$ Glc: $X=O A c Y=H$

2d = Gal: $X=H \quad Y=O A c$

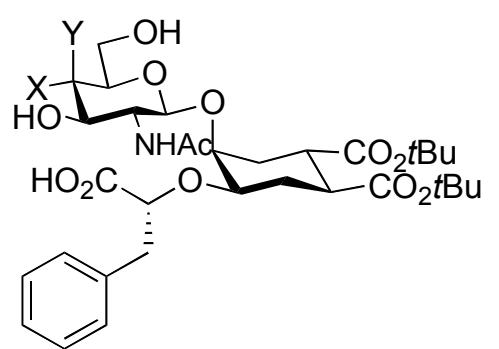

$2 \mathrm{e}=$ GIcNAc: $\mathrm{X}=\mathrm{OAc} \mathrm{Y}=\mathrm{H}$

2f $=$ GalNAc: $X=H Y=O A c$

To a solution of $9 \mathrm{c}-9 \mathbf{f}(1 \mathrm{eq})$ in dry $\mathrm{MeOH}(0.05 \mathrm{M})$, under $\mathrm{N}_{2}$, a solution $1 \mathrm{M} \mathrm{MeONa}$ in $\mathrm{MeOH}(1.5 \mathrm{eq})$ was added. The reaction was stirred at room temperature monitoring by TLC $\left(\mathrm{CHCl}_{3} / \mathrm{MeOH} 90: 10\right.$ and $\left.2 \% \mathrm{H}_{2} \mathrm{O}\right)$. After completion, the reaction was quenched adding the resin Amberlite IR $50\left(\mathrm{H}^{+}\right.$form) until $\mathrm{pH}=5-6$ was reached. Finally the resin was filtered and washed several time with $\mathrm{MeOH}$. The organic phase was evaporated under reduced pressure and the crude was purified by flash chromatography on silica gel. The products $\mathbf{2 c - 2 f}$ were obtained as amorphous white solid.

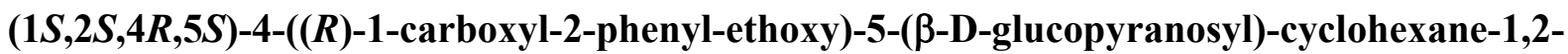
dicarboxyl acid di-tert-butyl ester (2c):

Chromatography eluent: $\left(\mathrm{CHCl}_{3} / \mathrm{MeOH}: 85: 15\right.$ and $\left.2 \% \mathrm{H}_{2} \mathrm{O}\right)$, (yield: $\left.7 \mathrm{mg}, 70 \%\right)$; $[\alpha]^{25}{ }_{\mathrm{D}}=-3.11 \mathrm{c}=0.45$ $\mathrm{CHCl}_{3} ;{ }^{1} \mathrm{H}-\mathrm{NMR}\left(\mathrm{D}_{2} \mathrm{O}, 400 \mathrm{MHz}\right): 1.35\left(\mathrm{~s}, 9 \mathrm{H},\left(\mathrm{CH}_{3}\right)_{3} \mathrm{C}-\right), 1.38\left(\mathrm{~s}, 9 \mathrm{H},\left(\mathrm{CH}_{3}\right)_{3} \mathrm{C}-\right), 1.40-1.45(\mathrm{~m}, 1 \mathrm{H}$, $\left.\mathrm{H}_{6 \mathrm{ax}}\right), 1.75$ (ddd, $\left.1 \mathrm{H}, \mathrm{H}_{3 \mathrm{ax}}, \mathrm{J}_{\mathrm{gem}}=\mathrm{J}_{3 \mathrm{ax}-2}=\mathrm{J}_{3 \mathrm{ax}-4}=12 \mathrm{~Hz}\right), 2.00-2.10\left(\mathrm{~m}, 1 \mathrm{H}, \mathrm{H}_{3 \mathrm{eq}}\right), 2.18\left(\mathrm{dt}, 1 \mathrm{H}, \mathrm{H}_{6 \mathrm{eq}}, \mathrm{J}_{\text {gem }}=\right.$ $14 \mathrm{~Hz}$ and $\left.\mathrm{J}_{6 \mathrm{eq}-1}=\mathrm{J}_{6 \mathrm{eq}-5}=3.7 \mathrm{~Hz}\right), 2.5\left(\mathrm{td}, 1 \mathrm{H}, \mathrm{H}_{2}, \mathrm{~J}_{2-3 \mathrm{ax}}=\mathrm{J}_{2-1}=13 \mathrm{~Hz}\right.$ and $\left.\mathrm{J}_{2-3 \mathrm{eq}}=3.7 \mathrm{~Hz}\right), 2.74\left(\mathrm{td}, 1 \mathrm{H}, \mathrm{H}_{1}\right.$, $\mathrm{J}_{1-6 \mathrm{ax}}=\mathrm{J}_{1-2}=13$ and $\left.\mathrm{Hz} \mathrm{J}_{1-6 \mathrm{eq}}=3.7 \mathrm{~Hz}\right), 2.88\left(\mathrm{dd}, 1 \mathrm{H}, \mathrm{CHHPh}, \mathrm{J}_{\mathrm{gem}}=14 \mathrm{~Hz}\right.$ and $\left.\mathrm{J}_{\mathrm{CHPh}-\mathrm{HL}}=8 \mathrm{~Hz}\right), 3.02$ (dd, $1 \mathrm{H}, \mathrm{CH} H \mathrm{Ph}, \mathrm{J}_{\mathrm{gem}}=14 \mathrm{~Hz}$ and $\left.\mathrm{J}_{\mathrm{CHPh}-\mathrm{HL}}=5 \mathrm{~Hz}\right), 3.10-3.20\left(\mathrm{~m}, 2 \mathrm{H}, \mathrm{GlcH}_{5}\right.$ and $\left.\mathrm{GlcH}_{2}\right), 3.25-3.39(\mathrm{~m}, 3 \mathrm{H}$, $\mathrm{GlcH}_{3}, \mathrm{GlcH}_{4}$ and $\left.\mathrm{H}_{4}\right), 3.65\left(\mathrm{dd}, 1 \mathrm{H}, \mathrm{GlcH}_{6}, \mathrm{~J}_{\mathrm{gem}}=12 \mathrm{~Hz}\right.$ and $\left.\mathrm{J}_{\mathrm{GH} 6-\mathrm{GH}}=5.6 \mathrm{~Hz}\right), 3.82\left(\mathrm{dd}, 1 \mathrm{H}, \mathrm{GlcH}_{6}\right.$, $\mathrm{J}_{\mathrm{gem}}=12 \mathrm{~Hz}$ and $\left.\mathrm{J}_{\mathrm{GH} 6-\mathrm{GH} 5}=2 \mathrm{~Hz}\right), 3.97\left(\mathrm{bs}, 1 \mathrm{H}, \mathrm{H}_{5}\right), 4.07\left(\mathrm{~d}, 1 \mathrm{H}, \mathrm{GlcH}_{1}, \mathrm{~J}_{\mathrm{GH} 1-\mathrm{GH} 2}=8 \mathrm{~Hz}\right), 4.20(\mathrm{dd}, 1 \mathrm{H}$, $\mathrm{H}_{\mathrm{L}}, \mathrm{J}_{\mathrm{HL}-\mathrm{CH} 2 \mathrm{Ph}}=8$ and $\left.5 \mathrm{~Hz}\right), 7.20-7.40\left(\mathrm{~m}, 5 \mathrm{H}, \mathrm{H}_{\mathrm{ar}}\right) \mathrm{ppm} ;{ }^{13} \mathrm{C}-\mathrm{NMR}\left(\mathrm{D}_{2} \mathrm{O}, 100.6 \mathrm{MHz}\right): 27.16,27.18$, 28.36, 32.69, 39.24, 40.21, 44.51, 60.82, 69.61, 73.45, 73.96, 75.58, 75.63, 78.60, 81.68, 83.00, 83.18, $102.62,126.78,128.68,129.46,138.12,175.43$ ( 2 x C), 176.38 ppm; HRMS (ESI) $\mathrm{C}_{31} \mathrm{H}_{46} \mathrm{O}_{13}$ calcd. $[\mathrm{M}-\mathrm{H}]^{-}: 625.28657$ found $[\mathrm{M}-\mathrm{H}]^{-}: 625.28500$

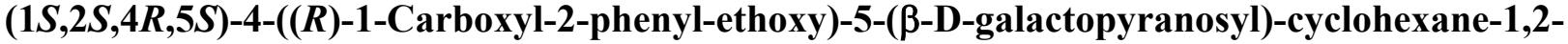 dicarboxyl acid di-tert-butyl ester (2d):}

Chromatography eluent: $\left(\mathrm{CHCl}_{3} / \mathrm{MeOH}: 85: 15\right.$ and $\left.2 \% \mathrm{H}_{2} \mathrm{O}\right)$, (yield: $\left.12 \mathrm{mg}, 75 \%\right),[\alpha]^{25}=+9.55 \mathrm{c}=0.9$ $\mathrm{CHCl}_{3} ;{ }^{1} \mathrm{H}-\mathrm{NMR}\left(\mathrm{D}_{2} \mathrm{O}, 400 \mathrm{MHz}\right): 1.35\left(\mathrm{~s}, 9 \mathrm{H},\left(\mathrm{CH}_{3}\right)_{3} \mathrm{C}-\right), 1.38\left(\mathrm{~s}, 9 \mathrm{H},\left(\mathrm{CH}_{3}\right)_{3} \mathrm{C}-\right), 1.38-1.47(\mathrm{~m}, 1 \mathrm{H}$, $\left.\mathrm{H}_{6 \mathrm{ax}}\right), 1.77$ (ddd, $\left.1 \mathrm{H}, \mathrm{H}_{3 \mathrm{ax}}, \mathrm{J}_{\mathrm{gem}}=\mathrm{J}_{3 \mathrm{ax}-2}=\mathrm{J}_{3 \mathrm{ax}-4}=12 \mathrm{~Hz}\right), 2.00-2.10\left(\mathrm{~m}, 1 \mathrm{H}, \mathrm{H}_{3 \mathrm{eq}}\right), 2.16-2.24\left(\mathrm{~m}, 1 \mathrm{H}, \mathrm{H}_{6 \mathrm{eq}}\right)$, 2.48-2.52 (m, $\left.1 \mathrm{H}, \mathrm{H}_{2}\right), 2.70-2.80\left(\mathrm{~m}, 1 \mathrm{H}, \mathrm{H}_{1}\right), 2.87\left(\mathrm{dd}, 1 \mathrm{H}, \mathrm{CH} \mathrm{HPh}, \mathrm{J}_{\mathrm{gem}}=13 \mathrm{~Hz}\right.$ and $\left.\mathrm{J}_{\mathrm{CHPh}-\mathrm{HL}}=9 \mathrm{~Hz}\right)$, $2.98\left(\mathrm{dd}, 1 \mathrm{H}, \mathrm{CH} H \mathrm{Ph}, \mathrm{J}_{\mathrm{gem}}=13 \mathrm{~Hz}\right.$ and $\left.\mathrm{J}_{\mathrm{CHPh}-\mathrm{HL}}=4 \mathrm{~Hz}\right), 3.25-3.35\left(\mathrm{~m}, 2 \mathrm{H}, \mathrm{GalH}_{5}\right.$ and $\left.\mathrm{H}_{4}\right), 3.40-3.45$ $\left(\mathrm{m}, 2 \mathrm{H}, \mathrm{GalH}_{3}\right.$ and $\left.\mathrm{GalH}_{2}\right), 3.65-3.73\left(\mathrm{~m}, 2 \mathrm{H}, 2 \times \mathrm{GalH}_{6}\right), 3.80\left(\mathrm{bs}, 1 \mathrm{H}, \mathrm{GalH}_{4}\right), 3.97-4.06(\mathrm{~m}, 2 \mathrm{H}$, $\mathrm{GalH}_{1}$ and $\left.\mathrm{H}_{5}\right), 4.15-4.22\left(\mathrm{~m}, 1 \mathrm{H}, \mathrm{H}_{\mathrm{L}}\right), 7.20-7.40\left(\mathrm{~m}, 5 \mathrm{H}, \mathrm{H}_{\mathrm{ar}}\right) \mathrm{ppm} ;{ }^{13} \mathrm{C}-\mathrm{NMR}\left(\mathrm{D}_{2} \mathrm{O}, 50.3 \mathrm{MHz}\right): 25.63$, 26.85, 31.29, 37.71, 38.68, 43.01, 59.29, 66.98, 69.56, 71.05, 71. 96, 73.19, 77.12, 79.97, 81.52, 81.66, 101.59, 125.17, 127.16, 127.98, 136.57, 173.91, 174.93, 178.15 ppm; HRMS (ESI) $\mathrm{C}_{31} \mathrm{H}_{46} \mathrm{O}_{13}$ calcd. $[\mathrm{M}-\mathrm{H}]^{-}: 625.28657$ found $[\mathrm{M}-\mathrm{H}]^{-}: 625.28518$

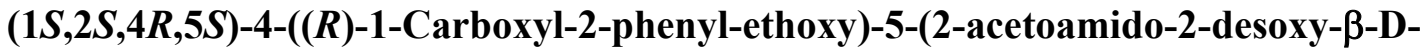
glucopyranosyl)-cyclohexane-1,2-dicarboxyl acid di-tert-butyl ester (2e):

(yield: $6 \mathrm{mg}, 90 \%),[\alpha]^{25}=-2.22 \mathrm{c}=0.45 \mathrm{CHCl}_{3} ;{ }^{1} \mathrm{H}-\mathrm{NMR}\left(\mathrm{D}_{2} \mathrm{O}, 400 \mathrm{MHz}\right): 1.36\left(\mathrm{~s}, 9 \mathrm{H},\left(\mathrm{CH}_{3}\right)_{3} \mathrm{C}-\right)$, 1.39 (s, 9H, $\left.\left(\mathrm{CH}_{3}\right)_{3} \mathrm{C}-\right), 1.40-1.50\left(\mathrm{~m}, 2 \mathrm{H}, \mathrm{H}_{3 \mathrm{ax}}\right.$ and $\left.\mathrm{H}_{6 \mathrm{ax}}\right), 1.90\left(\mathrm{~s}, 3 \mathrm{H}, \mathrm{CH}_{3} \mathrm{CONH}-\right), 2.00-2.10(\mathrm{~m}, 2 \mathrm{H}$, $\mathrm{H}_{3 \mathrm{eq}}$ and $\mathrm{H}_{6 \mathrm{eq}}$ ), 2.45 (td, $1 \mathrm{H}, \mathrm{H}_{2}, \mathrm{~J}_{2-3 \mathrm{ax}}=\mathrm{J}_{2-1}=12 \mathrm{~Hz}$ and $\left.\mathrm{J}_{2-3 \mathrm{eq}}=3 \mathrm{~Hz}\right), 2.60\left(\mathrm{td}, 1 \mathrm{H}, \mathrm{H}_{1}, \mathrm{~J}_{1-6 \mathrm{ax}}=\mathrm{J}_{1-2}=12 \mathrm{~Hz}\right.$ and $\left.\mathrm{J}_{1-6 \mathrm{eq}}=3 \mathrm{~Hz}\right), 2.90\left(\mathrm{dd}, 1 \mathrm{H}, \mathrm{CHHPh}, \mathrm{J}_{\mathrm{gem}}=14\right.$ and $\left.\mathrm{Hz} \mathrm{J}_{\mathrm{CHPh}-\mathrm{HL}}=7 \mathrm{~Hz}\right), 3.02-3.20(\mathrm{~m}, 3 \mathrm{H}, \mathrm{CH} H \mathrm{Ph}$, 
$\mathrm{GlcNAcH}_{3}$ and $\left.\mathrm{GlcNAcH}_{5}\right), 3.25-3.40\left(\mathrm{~m}, 2 \mathrm{H}, \mathrm{GlcNAcH}_{4}\right.$ and $\left.\mathrm{H}_{4}\right) 3.58\left(\mathrm{t}, 1 \mathrm{H}, \mathrm{GlcNAcH}_{2}, \mathrm{~J}_{\mathrm{GH} 2-}\right.$ $\left.\mathrm{GH}_{1}=\mathrm{J}_{\mathrm{GH} 2-\mathrm{GH} 3}=9 \mathrm{~Hz}\right), 3.65\left(\mathrm{dd}, 1 \mathrm{H}, \mathrm{GlcNAcH}_{6}, \mathrm{~J}_{\mathrm{gem}}=12 \mathrm{~Hz}\right.$ and $\left.\mathrm{J}_{\mathrm{GH} 6-\mathrm{GH} 5}=5.6 \mathrm{~Hz}\right), 3.80-3.87(\mathrm{~m}, 1 \mathrm{H}$, $\left.\mathrm{GlcNAcH}_{6}\right), 4.05\left(\mathrm{bs}, 1 \mathrm{H}, \mathrm{H}_{5}\right), 4.15-4.22\left(\mathrm{~m}, 1 \mathrm{H}, \mathrm{H}_{\mathrm{L}}\right), 4.48\left(\mathrm{~d}, 1 \mathrm{H}, \mathrm{GlcNAcH}_{1}, \mathrm{~J}_{\mathrm{GH} 1-\mathrm{GH} 2}=9 \mathrm{~Hz}\right), 7.20-$ $7.40\left(\mathrm{~m}, 5 \mathrm{H}, \mathrm{H}_{\mathrm{ar}}\right) \mathrm{ppm} ;{ }^{13} \mathrm{C}-\mathrm{NMR}\left(\mathrm{D}_{2} \mathrm{O}, 100.6 \mathrm{MHz}\right): 22.38,27.11,27.16,28.20,32.64,38.69,40.22$, $44.41,55.53,60.76,69.86,72.97,73.98,75.79,77.79,80.08,82.94,82.96,100.55,126.87,128.68$, 129.59, 138.31, 174.18, 175.14 ( 2 x C), 176.16 ppm; HRMS (ESI) $\mathrm{C}_{33} \mathrm{H}_{49} \mathrm{NO}_{13}$ calcd. [M-H]: 666.31311 found $[\mathrm{M}-\mathrm{H}]^{-}: 666.31118$

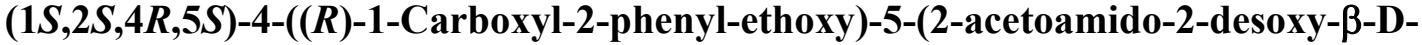
galactopyranosyl)-cyclohexane-1,2-dicarboxyl acid di-tert-butyl ester (2f):

Chromatography eluent: $\left(\mathrm{CHCl}_{3} / \mathrm{MeOH}\right.$ : 85:15 and $\left.2 \% \mathrm{H}_{2} \mathrm{O}\right)$, (yield: $\left.11 \mathrm{mg}, 87 \%\right),[\alpha]^{25}=1.66 \mathrm{c}=0.9$ $\mathrm{CHCl}_{3} ;{ }^{1} \mathrm{H}-\mathrm{NMR}\left(\mathrm{D}_{2} \mathrm{O}, 400 \mathrm{MHz}\right): 1.40\left(\mathrm{~s}, 9 \mathrm{H},\left(\mathrm{CH}_{3}\right)_{3} \mathrm{C}-\right), 1.45\left(\mathrm{~s}, 9 \mathrm{H},\left(\mathrm{CH}_{3}\right)_{3} \mathrm{C}-\right), 1.45-1.60$ (m, 2H, $\mathrm{H}_{3 \mathrm{ax}}$ and $\left.\mathrm{H}_{6 \mathrm{ax}}\right), 1.98$ (s, 3H, $\mathrm{CH}_{3} \mathrm{CONH}-$ ), 2.05-2.15 (m, $2 \mathrm{H}, \mathrm{H}_{3 \mathrm{eq}}$ and $\mathrm{H}_{6 \mathrm{eq}}$ ), 2.47 (td, $1 \mathrm{H}, \mathrm{H}_{2}, \mathrm{~J}_{2-3 \mathrm{ax}}=\mathrm{J}_{2-1}=$ $11 \mathrm{~Hz}$ and $\left.\mathrm{J}_{2-3 \mathrm{eq}}=3.5 \mathrm{~Hz}\right), 2.65\left(\mathrm{td}, 1 \mathrm{H}, \mathrm{H}_{1}, \mathrm{~J}_{1-6 \mathrm{ax}}=\mathrm{J}_{1-2}=11 \mathrm{~Hz}\right.$ and $\left.\mathrm{J}_{1-6 \mathrm{eq}}=3.5 \mathrm{~Hz}\right), 2.95(\mathrm{dd}, 1 \mathrm{H}, \mathrm{CHHPh}$, $\mathrm{J}_{\mathrm{gem}}=14 \mathrm{~Hz}$ and $\left.\mathrm{J}_{\mathrm{CHPh}-\mathrm{HL}}=7 \mathrm{~Hz}\right), 3.10\left(\mathrm{dd}, 1 \mathrm{H}, \mathrm{CH} H \mathrm{Ph}, \mathrm{J}_{\mathrm{gem}}=14 \mathrm{~Hz}\right.$ and $\left.\mathrm{J}_{\mathrm{CHPh}-\mathrm{HL}}=4 \mathrm{~Hz}\right), 3.28-3.45(\mathrm{~m}$, $3 \mathrm{H}, \mathrm{H}_{4}, \mathrm{GalNAcH}_{3}$ and $\left.\mathrm{GalNAcH}_{5}\right), 3.70-3.80\left(\mathrm{~m}, 2 \mathrm{H}, 2 \mathrm{x} \mathrm{GalNAcH}_{6}\right), 3.82-3.90\left(\mathrm{~m}, 2 \mathrm{H}, \mathrm{GalNAcH}_{2}\right.$ and $\left.\mathrm{GalNAcH}_{4}\right), 4.10\left(\mathrm{bs}, 1 \mathrm{H}, \mathrm{H}_{5}\right), 4.27\left(\mathrm{dd}, 1 \mathrm{H}, \mathrm{H}_{\mathrm{L}}, \mathrm{J}_{\mathrm{HL}-\mathrm{CH} 2 \mathrm{Ph}}=7\right.$ and $\left.4 \mathrm{~Hz}\right), 4.07\left(\mathrm{~d}, 1 \mathrm{H}, \mathrm{GalNAcH}_{1}\right.$, $\left.\mathrm{J}_{\mathrm{GH} 1-\mathrm{GH} 2}=9 \mathrm{~Hz}\right), 7.30-7.45\left(\mathrm{~m}, 5 \mathrm{H}, \mathrm{H}_{\mathrm{ar}}\right) \mathrm{ppm} ;{ }^{13} \mathrm{C}-\mathrm{NMR}\left(\mathrm{D}_{2} \mathrm{O}, 50.3 \mathrm{MHz}\right): 20.92,25.60,26.28,31.12$, $37.14,38.63,42.88,59.25,66.22,69.70,71.08,73.44,76.55,78.26,81.39,99.48,125.30,127.15$, 128.07, 136.60, 172.82, 173.57, 174.64, 177.86 ppm; HRMS (ESI) $\mathrm{C}_{33} \mathrm{H}_{49} \mathrm{NO}_{13}$ calcd. [M-H]: 666.31311 found $[\mathrm{M}-\mathrm{H}]^{-}: 666.31138$ 
${ }^{1} \mathrm{H}-\mathrm{NMR}\left(400 \mathrm{MHz}, \mathrm{D}_{2} \mathrm{O}\right): \mathbf{2 a}$

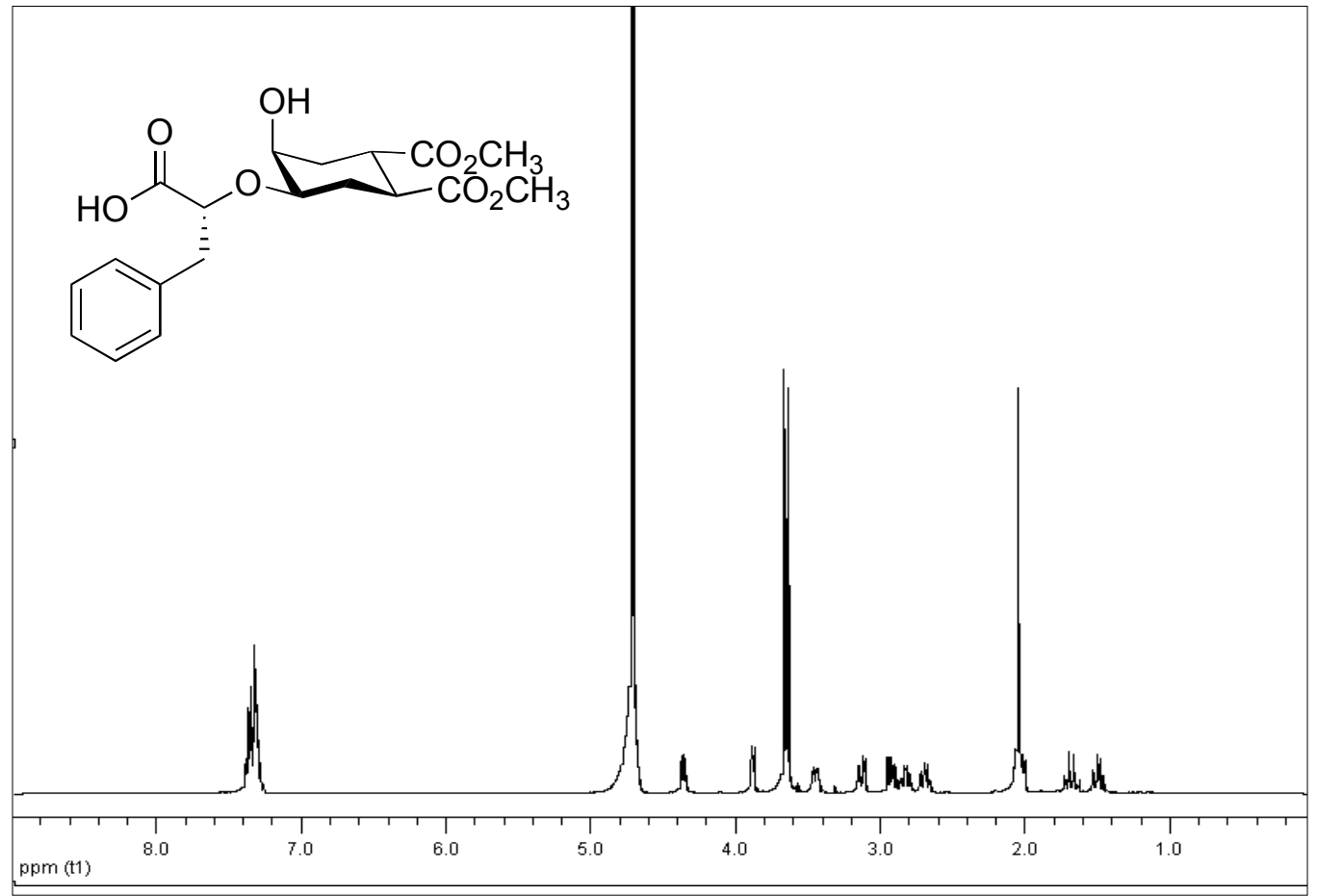

${ }^{13} \mathrm{C}-\mathrm{NMR}\left(50.3 \mathrm{MHz}, \mathrm{D}_{2} \mathrm{O}\right): \mathbf{2 a}$

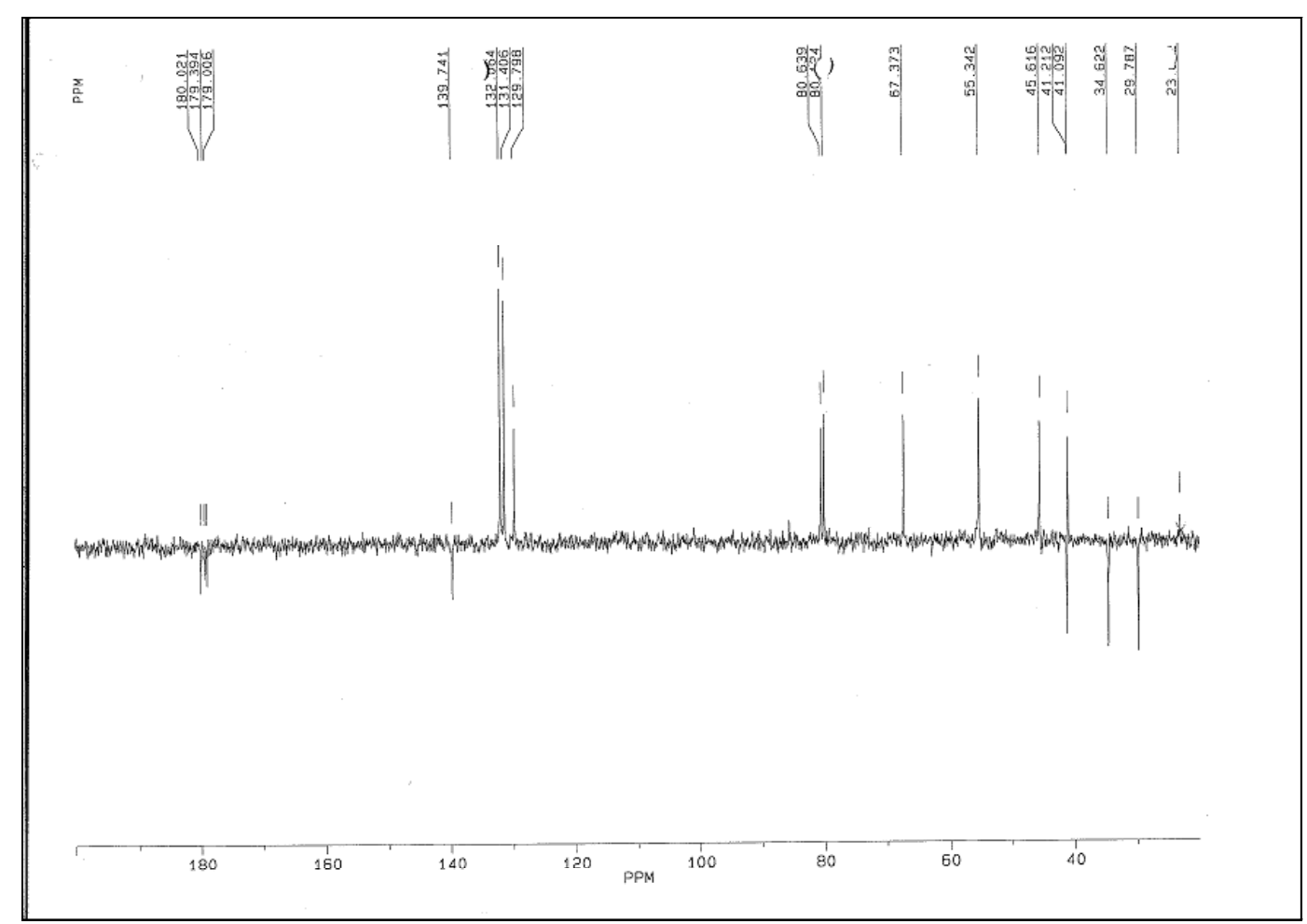


${ }^{1} \mathrm{H}-\mathrm{NMR}\left(400 \mathrm{MHz}, \mathrm{D}_{2} \mathrm{O}\right): \mathbf{2 b}$

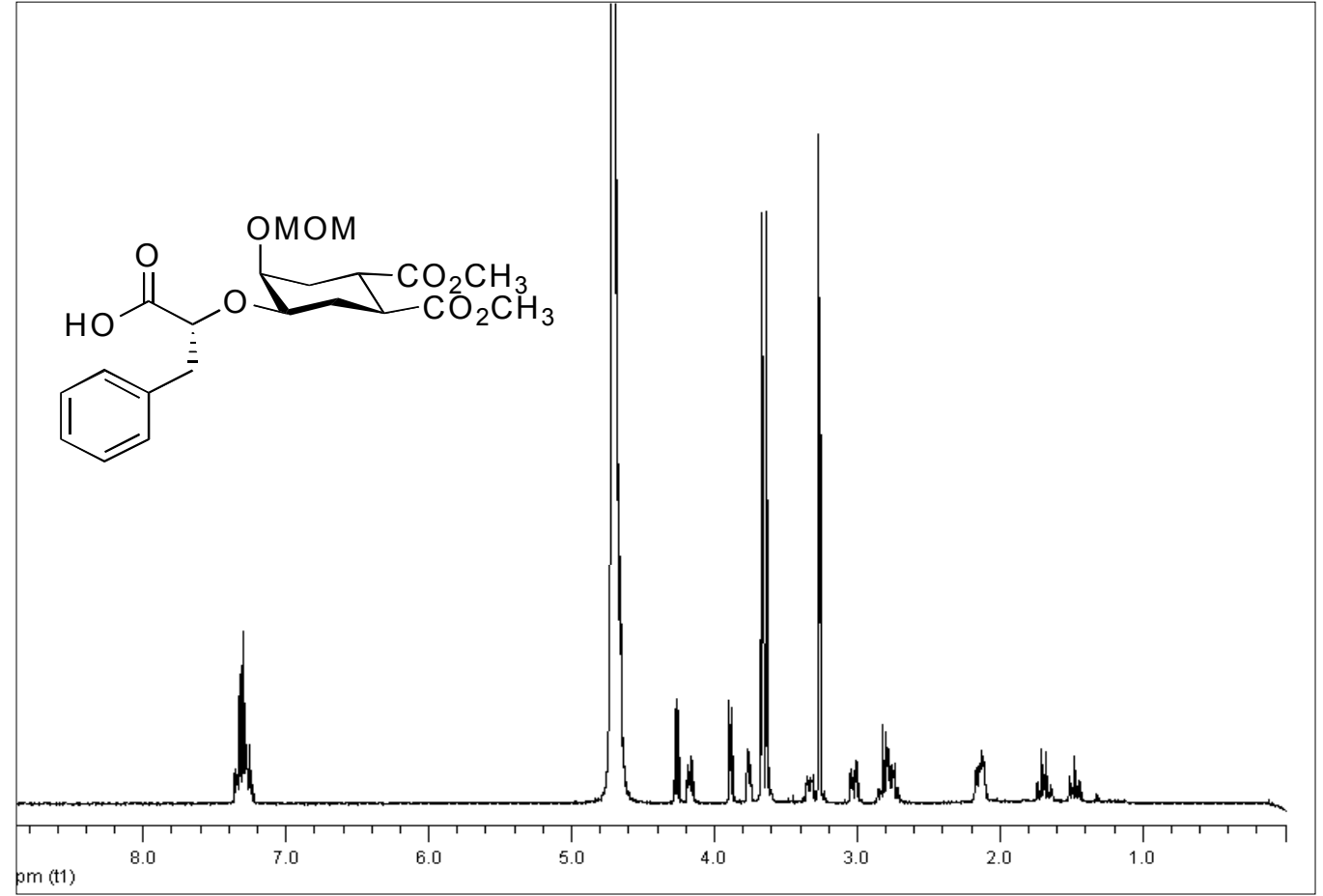

${ }^{13} \mathrm{C}-\mathrm{NMR}\left(100.6 \mathrm{MHz}, \mathrm{D}_{2} \mathrm{O}\right): \mathbf{2 b}$

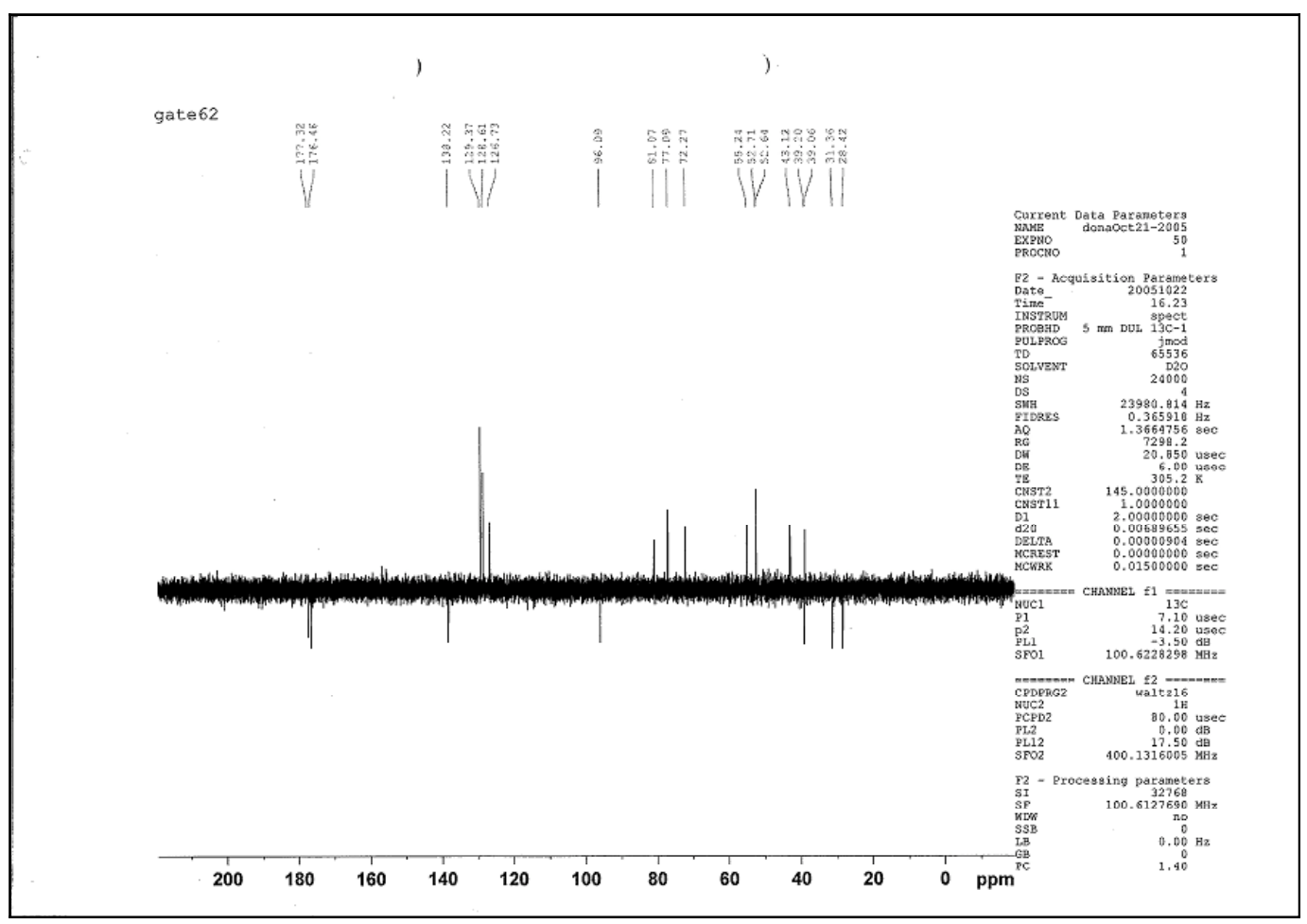


${ }^{1} \mathrm{H}-\mathrm{NMR}\left(400 \mathrm{MHz}, \mathrm{D}_{2} \mathrm{O}\right): \mathbf{2 c}$

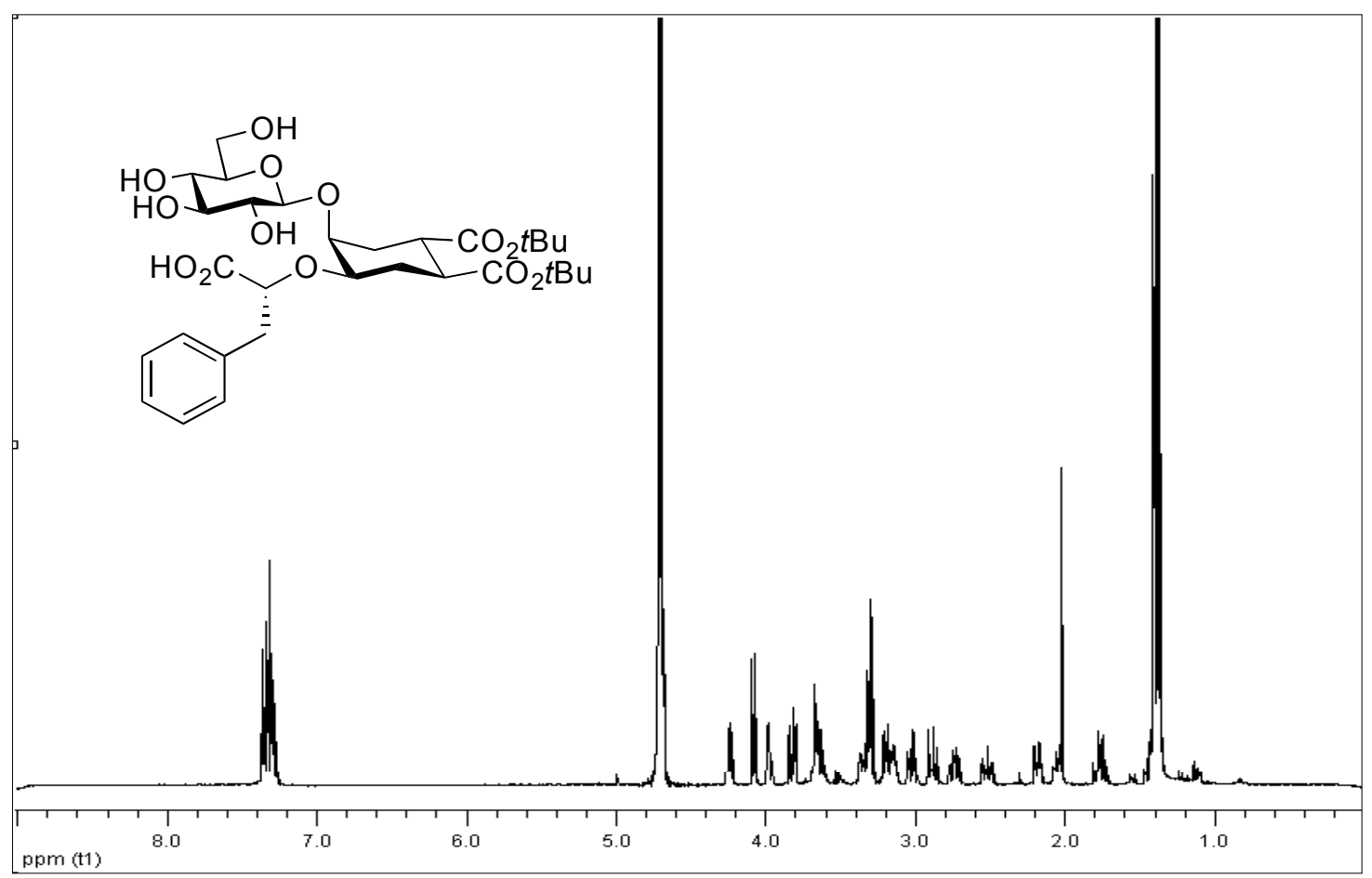

${ }^{13} \mathrm{C}-\mathrm{NMR}\left(100.6 \mathrm{MHz}, \mathrm{D}_{2} \mathrm{O}\right): \mathbf{2 c}$

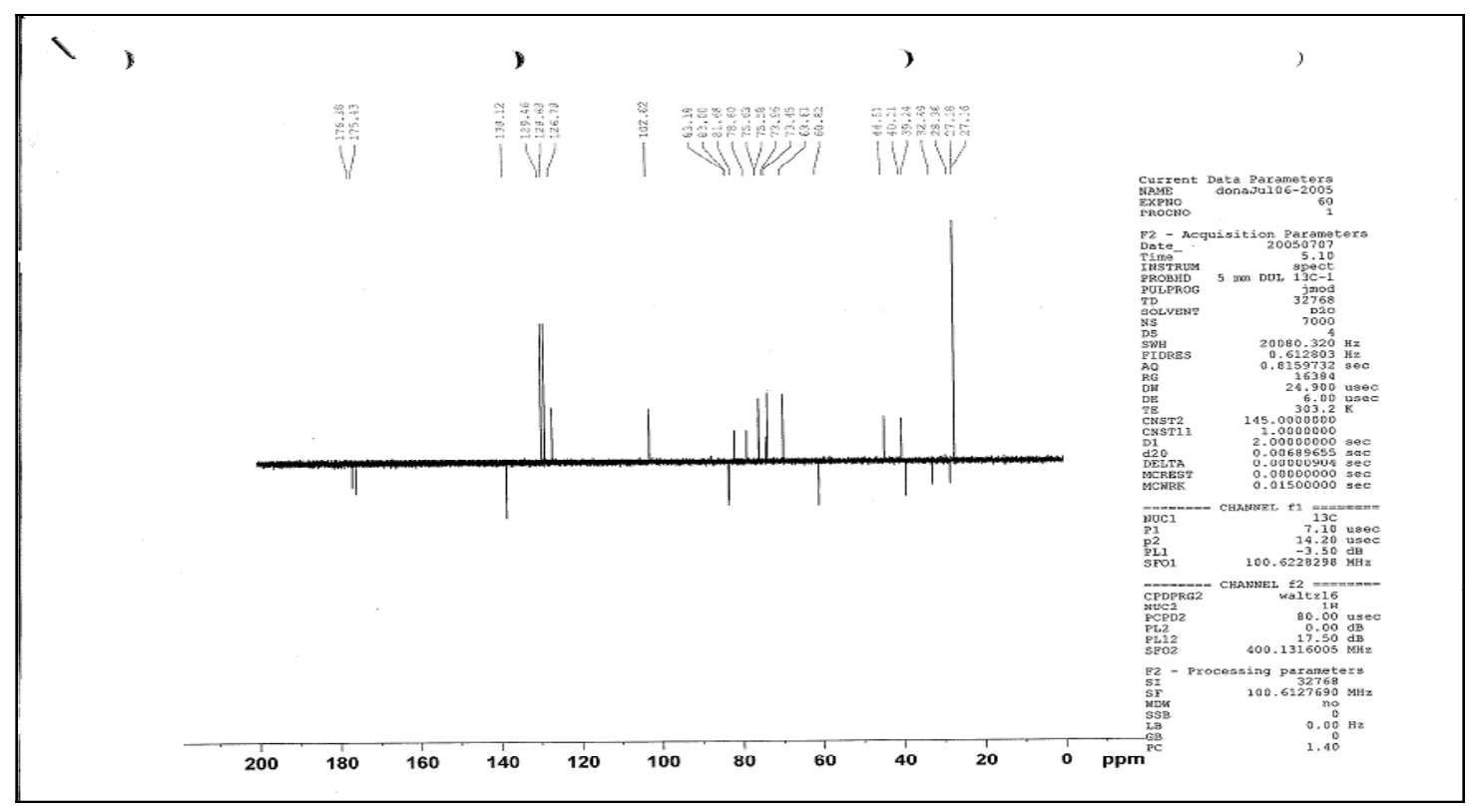


${ }^{1} \mathrm{H}-\mathrm{NMR}\left(400 \mathrm{MHz}, \mathrm{D}_{2} \mathrm{O}\right): \mathbf{2 d}$

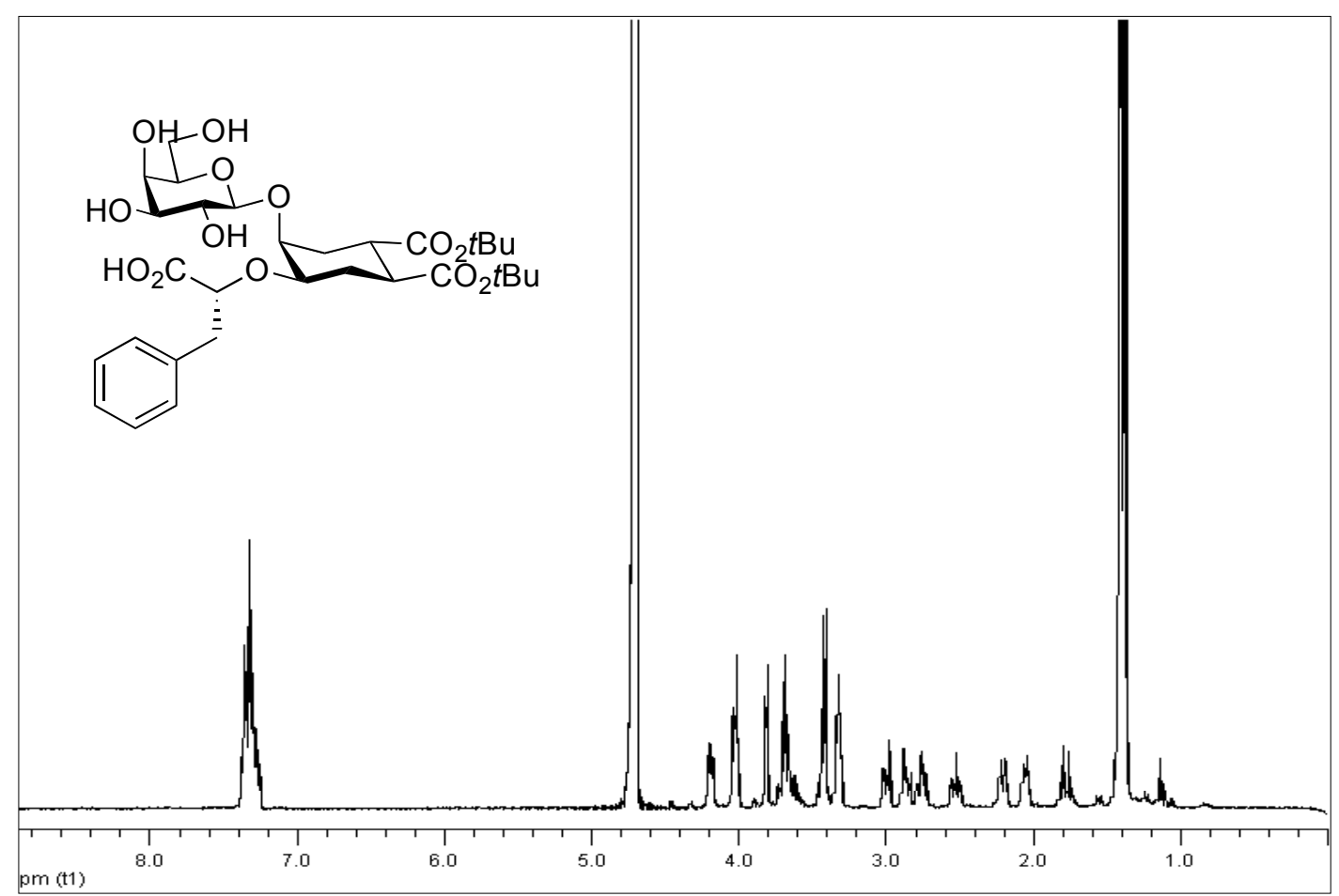

${ }^{13} \mathrm{C}-\mathrm{NMR}\left(50.3 \mathrm{MHz}, \mathrm{D}_{2} \mathrm{O}\right): 2 \mathbf{d}$ 


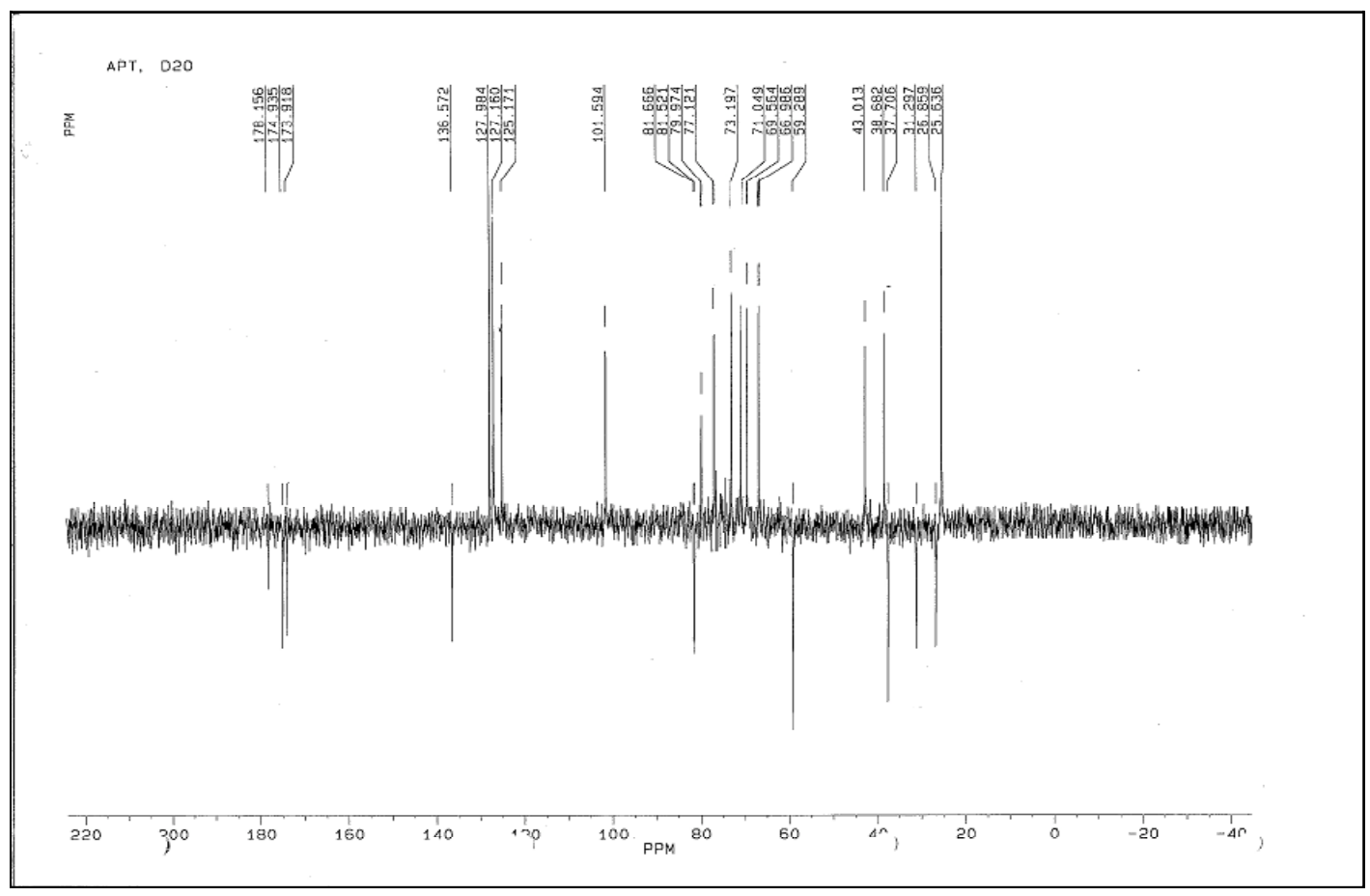

${ }^{1} \mathrm{H}-\mathrm{NMR}\left(400 \mathrm{MHz}, \mathrm{D}_{2} \mathrm{O}\right.$ ): $2 \mathbf{e}$

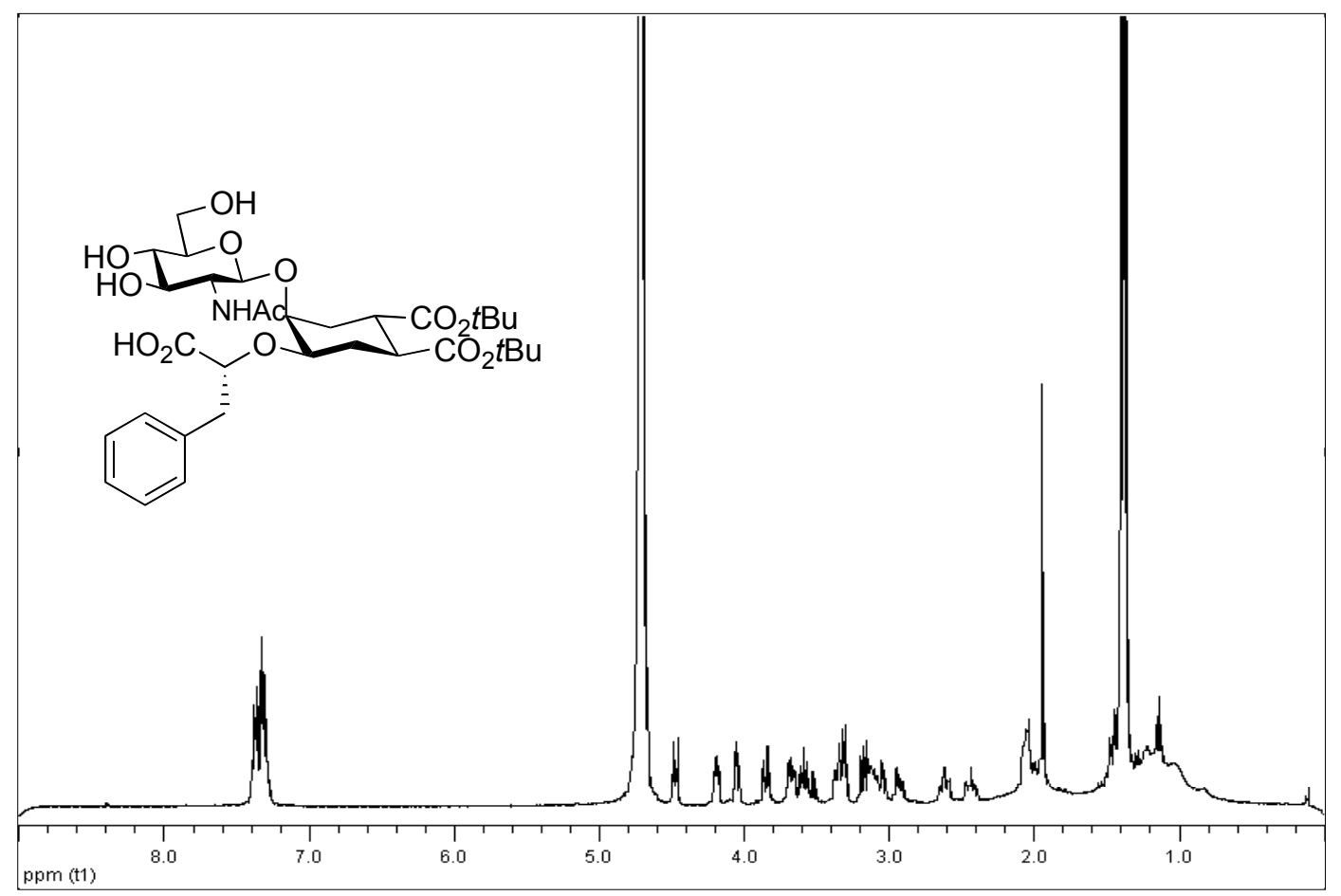


${ }^{13} \mathrm{C}-\mathrm{NMR}\left(100.6 \mathrm{MHz}, \mathrm{D}_{2} \mathrm{O}\right): \mathbf{2 e}$

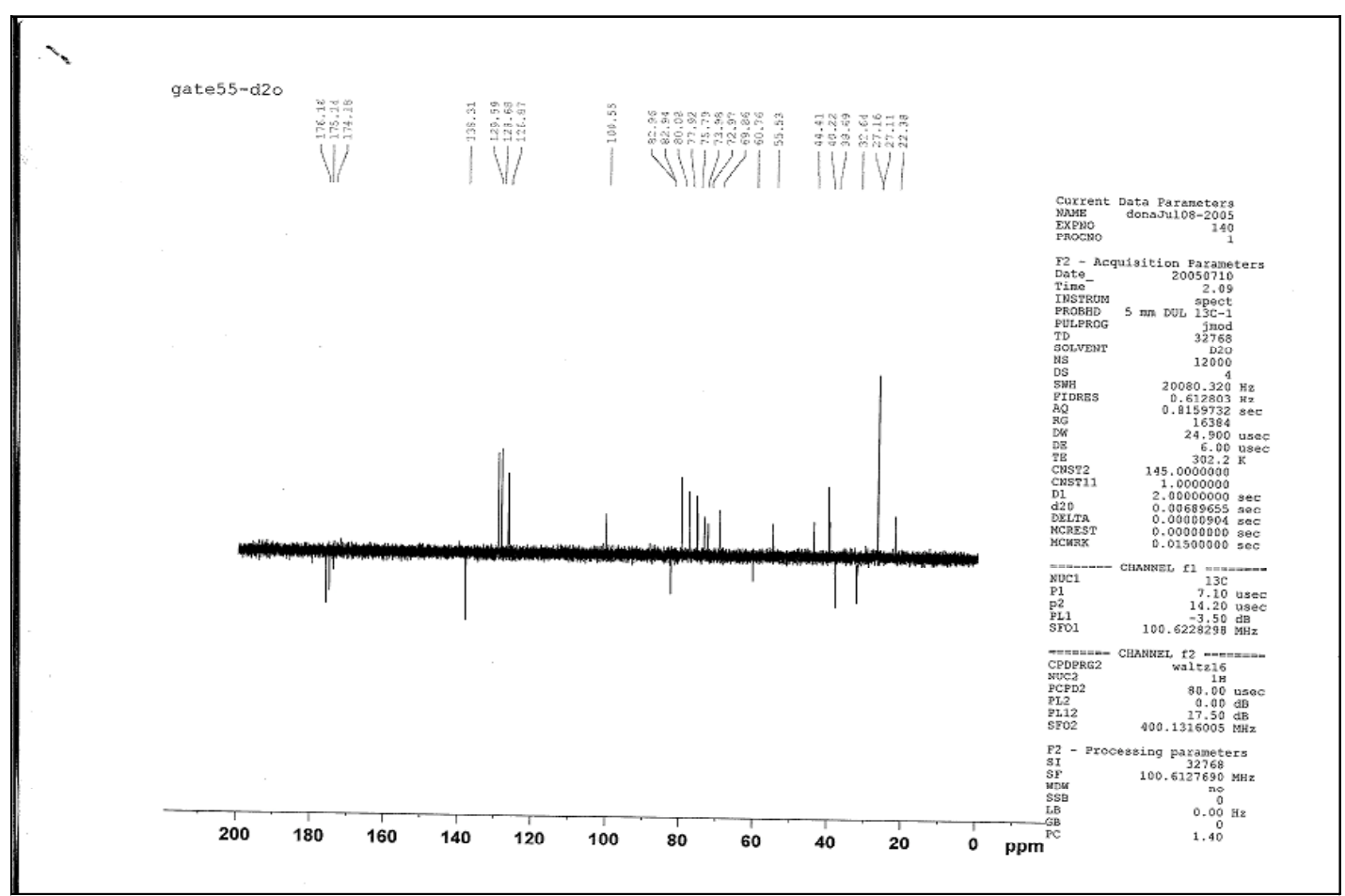

${ }^{1} \mathrm{H}-\mathrm{NMR}\left(400 \mathrm{MHz}, \mathrm{D}_{2} \mathrm{O}\right): \mathbf{2 f}$

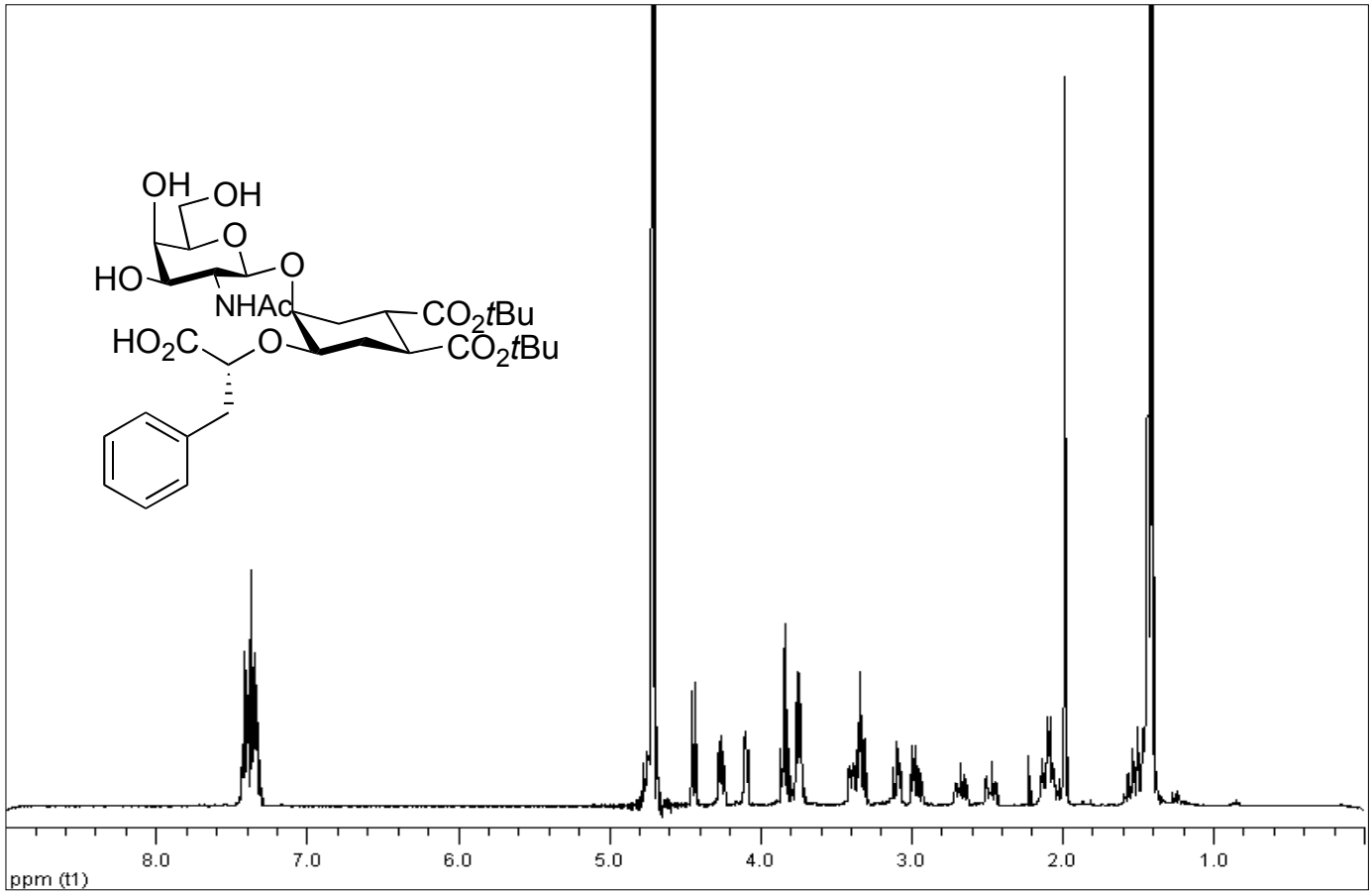


${ }^{13} \mathrm{C}-\mathrm{NMR}\left(50.3 \mathrm{MHz}, \mathrm{D}_{2} \mathrm{O}\right): \mathbf{2 f}$

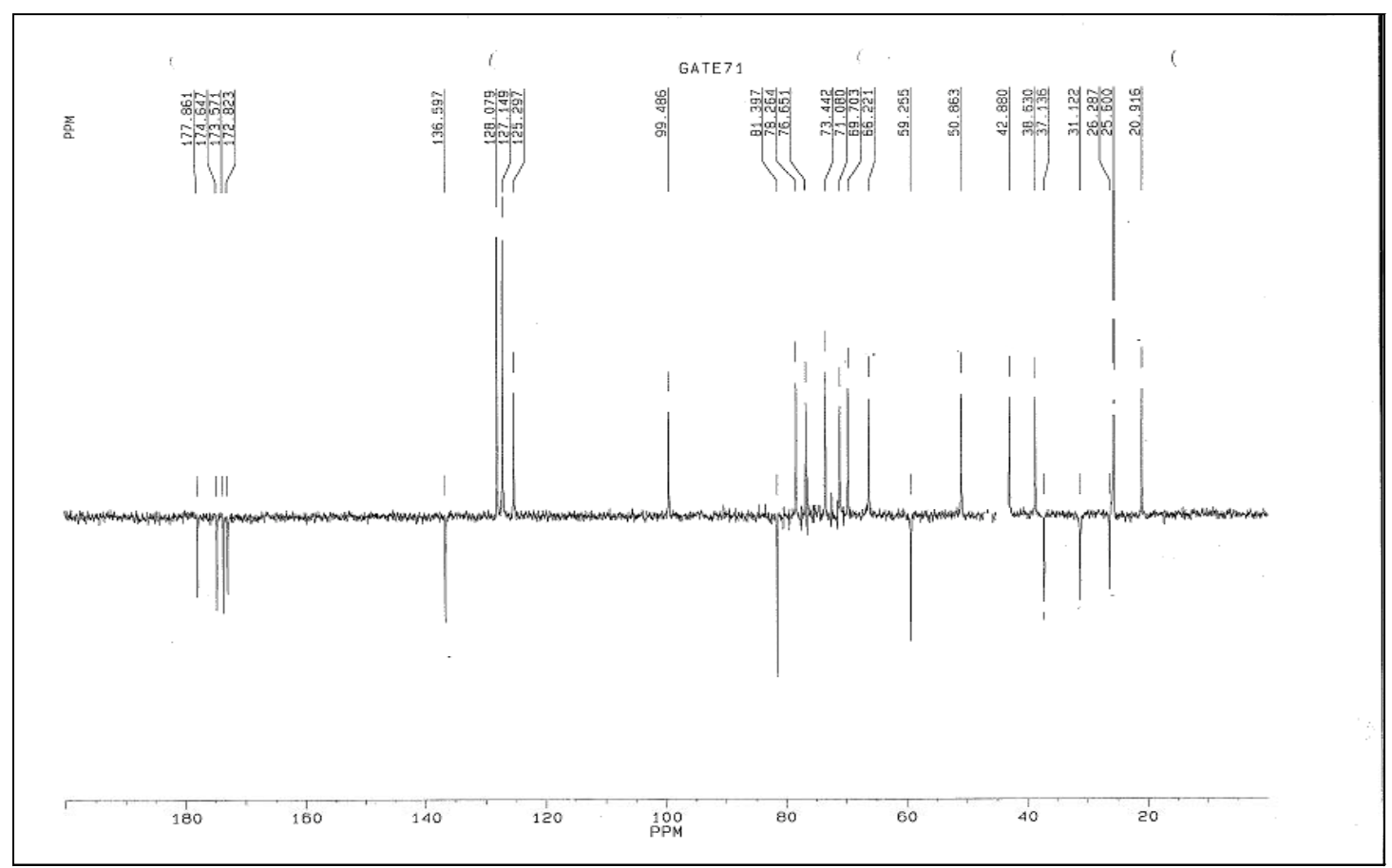

\section{References}

${ }^{1}$ Bernardi, A.; Boschin, G.; Checchia, A.; Lattanzio, M.; Manzoni, L.; Potenza, D.; Scolastico C. Eur. J. Org. Chem. 1999, 6, 1311-1317

${ }^{2}$ David, S.; Hanessian, S. Tetrahedrom 1985, 41, 643-649

${ }^{3}$ Bernardi, A.; Arosio, A.; Potenza, D.; Sanchez-Medina, I.; Mari, S.; Canada, F.J. Jiménez-Barbero, J.; Chem. Eur. J. 2004, 10, 4395-4406

${ }^{4}$ Danishefsky, S.; Hungate, R. J. Am. Chem. Soc. 1986, 108, 9, 2486-2487

${ }^{5}$ Stork, G.; Takahashi, T. J. Am. Chem. Soc. 1977, 99, 1275-1276

${ }^{6}$ Bernardi, A.; Boschin, G.; Checchia, A.; Lattanzio, M.; Manzoni, L.; Potenza, D.; Scolastico C. Eur. J. Org. Chem. 1999, 6, 1311-1317

${ }^{7}$ Zemplén, G. Ber. Dtsch. Chem. Ges. 1927, 60, 1555-1564. 